\title{
An Implicit Updated Lagrangian Formulation for Liquids with Large Surface Energy
}

\author{
DAVID A. B. HYDE, UCLA, USA \\ STEVEN W. GAGNIERE, UCLA, USA \\ ALAN MARQUEZ-RAZON, UCLA, USA \\ JOSEPH TERAN, UCLA, USA
}

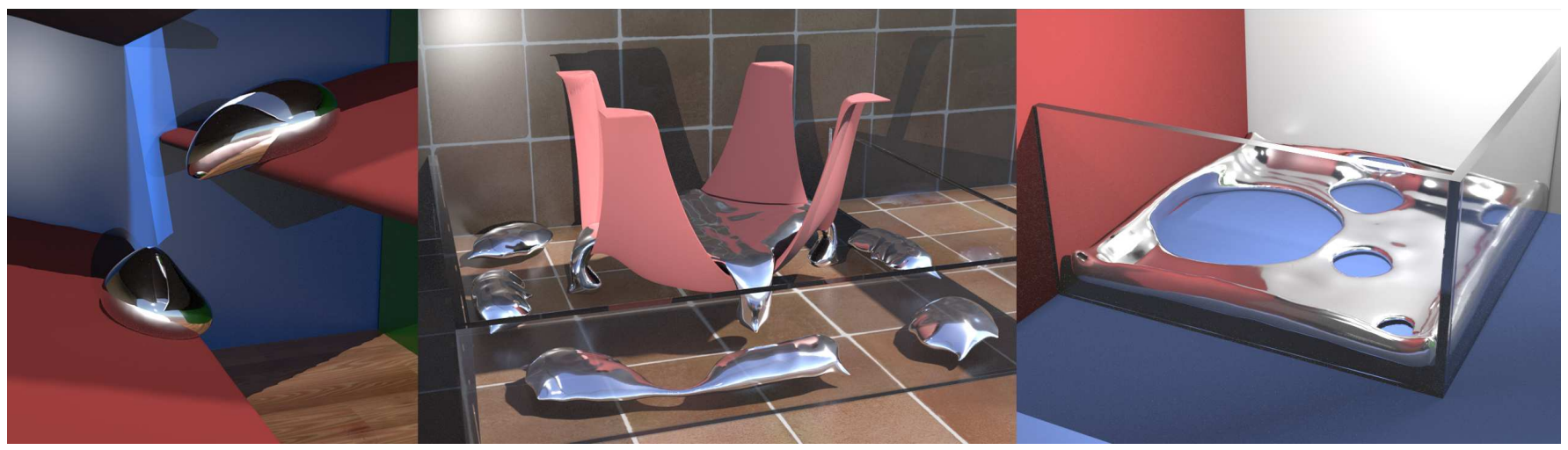

Fig. 1. Our numerical method enables the simulation of liquids with high surface energy, such as liquid mercury. Dynamic topology changes (left, middle, right) are naturally handled, and no special treatment is required to support one- and two-way coupling (left, middle).

We present an updated Lagrangian discretization of surface tension forces for the simulation of liquids with moderate to extreme surface tension effects. The potential energy associated with surface tension is proportional to the surface area of the liquid. We design discrete forces as gradients of this energy with respect to the motion of the fluid over a time step. We show that this naturally allows for inversion of the Hessian of the potential energy required with the use of Newton's method to solve the systems of nonlinear equations associated with implicit time stepping. The rotational invariance of the surface tension energy makes it non-convex and we define a definiteness fix procedure as in [Teran et al. 2005]. We design a novel level-set-based boundary quadrature technique to discretize the surface area calculation in our energy based formulation. Our approach works most naturally with Particle-In-Cell [Harlow 1964] techniques and we demonstrate our approach with a weakly incompressible model for liquid discretized with the Material Point Method [Sulsky et al. 1994]. We show that our approach is essential for allowing efficient implicit numerical integration in the limit of high surface tension materials like liquid metals.

CCS Concepts: • Mathematics of computing $\rightarrow$ Discretization; Partial differential equations; Solvers; • Applied computing $\rightarrow$ Physics.

Authors' addresses: David A. B. Hyde, UCLA, Box 951555, Los Angeles, CA, 90095-1555 USA, dabh@math.ucla.edu; Steven W. Gagniere, UCLA, Box 951555, Los Angeles, CA 90095-1555, USA, sgagniere@math.ucla.edu; Alan Marquez-Razon, UCLA, Box 951555 Los Angeles, CA, 90095-1555, USA, marqueza04@g.ucla.edu; Joseph Teran, UCLA, Box 951555, Los Angeles, CA, 90095-1555, USA, jteran@math.ucla.edu.

Permission to make digital or hard copies of part or all of this work for personal or classroom use is granted without fee provided that copies are not made or distributed for profit or commercial advantage and that copies bear this notice and the full citation on the first page. Copyrights for third-party components of this work must be honored. For all other uses, contact the owner/author(s).

(C) 2020 Copyright held by the owner/author(s).

0730-0301/2020/12-ART183

https://doi.org/10.1145/3414685.3417845
Additional Key Words and Phrases: Surface Tension, Material Point Methods, FLIP, PIC

\section{ACM Reference Format:}

David A. B. Hyde, Steven W. Gagniere, Alan Marquez-Razon, and Joseph Teran. 2020. An Implicit Updated Lagrangian Formulation for Liquids with Large Surface Energy. ACM Trans. Graph. 39, 6, Article 183 (December 2020), 13 pages. https://doi.org/10.1145/3414685.3417845

\section{INTRODUCTION}

Surface tension forces are responsible for many of the most striking features of fluid motion. Whether it be small scale droplets of water beading [Da et al. 2016; Li et al. 2020; Thürey et al. 2010; Wojtan et al. 2010; Yang et al. 2016], tears of wine in a glass [Azencot et al. 2015], or dynamic thin walls of a bubble [Da et al. 2015; Zhu et al. 2014], surface tension effects are indispensable tools in modern computer graphics applications. However, materials with extremely large surface tensions have not been explored to a great extent. For example, mercury and other liquid metals exhibit large surface tensions and yet remarkably have small viscosities [Zhao et al. 2017]. This combination of properties gives these materials characteristic flowing and merging dynamics strongly dominated by surface tension. We develop a novel method for simulating these materials with a Particle-In-Cell (PIC) approach [Harlow 1964] since it naturally resolves the necessary topological changes with modest computational cost. In particular, we observe that the governing physics of these materials are effectively hyperelastic [Bonet and Wood 2008] and therefore we develop a Material Point Method (MPM) [Sulsky et al. 1994] approach since this naturally allows for discretizations based on the elastic potential energy. 
Surface tension arises from the directional biases of molecular cohesion at the interface between different liquids. This can be modeled in a continuum with potential energy proportionate to the interfacial surface area [Adamson and Gast 1967; Brackbill et al. 1992; Buscaglia and Ausas 2011]. We resolve single phases of these liquids and in this case the surface tension effect is equivalently expressed as a traction boundary condition proportionate to the mean curvature scaled outward unit normal. Much of the existing literature is based on discretizing the curvature weighted unit normals, with the Continuum Surface Force formulation (CSF) of Brackbill et al. [1992] being the most influential. Notably, CSF is defined for PIC techniques that do not inherently maintain a notion of liquid boundary. In contrast, techniques that resolve the liquid surface with an explicit mesh allow for natural estimation of mean curvature from mesh connectivity [Cohen-Steiner and Morvan 2003]. However, these techniques are generally more computationally expensive. We also mention the popular, explicit method of Enright et al. [2003], which imposes a free surface boundary condition incorporating a surface tension term based on curvature. Combining the secondorder accurate discretization of Gibou et al. [2002] (with ghost values as appropriate) with a particle level set [Enright et al. 2002] representation of fluids, Enright et al. [2003] are able to estimate curvature at the interface using standard central finite differences.

As is commonly done with hyperelastic materials, we define our discretization directly from the potential energy [Sifakis and Barbic 2012] which, with surface tension, is simply proportionate to the surface area. This simplified conception allows us to discretize surface tension forces using particles without the need for any mesh connectivity or explicit mean curvature estimation. Instead, we must track how area-weighted normals on the boundary of the domain evolve in an updated Lagrangian view [Belytschko et al. 2013] of the flow kinematics. We show this is comparatively simple and develop a novel surface area quadrature approach utilizing particle derived level sets as in [Boyd and Bridson 2012]. We note that Misztal et al [2013] and Clausen et al. [2013] also define their discrete surface tension forces from surface energy; however, they do so with the aid of an explicitly tracked surface mesh.

For liquids like water with moderate to low surface tension, it generally suffices to use explicit time integration of surface tension forces. However, since we are interested in supporting materials with large surface energies, we develop a fully implicit approach. In particular, in order for an explicit method to resolve the fastest capillary wave speeds, the time step restriction has been shown to be $\Delta t \leq \sqrt{\frac{\rho \Delta x^{3}}{2 \pi k^{\sigma}}}$ for surface tension coefficient $k^{\sigma}$ and fluid density $\rho$ [Brackbill et al. 1992; Denner and van Wachem 2015]. Therefore, an implicit time stepping approach is very important for efficiency. Notably, while the CSF formulation [Brackbill et al. 1992] is very effective for PIC techniques, it does not easily generalize to implicit time stepping. In fact although semi-implicit treatments of surface tension forces, e.g. using a quadratic fit to the surface tension energy [Bänsch 2001; Misztal et al. 2013; Zheng et al. 2015] or by approximating the surface tension with mean curvature flow [Eckstein et al. 2007; Hysing 2006; Schroeder et al. 2012; Sussman and Ohta 2009; Thürey et al. 2010], are relatively common, fewer works such as Zheng et al. [2015] and Jarauta et al. [2018] take a fully implicit approach. However, Jarauta et al. [2018] only consider static Lagrangian mesh topology and cannot resolve the necessary topological changes that occur during flow, and Zheng et al. [2015] require nontrivial effort to process topology changes since they seek a volume-conserving scheme. In a slightly different setting, a fullyimplicit approach for surface tension using triangle-mesh-based areas is found in Batty et al. [2012], which has some precedent in explicit techniques such as Wojtan and Turk [2008].

Implicit time stepping requires solution of a root finding (or equivalently minimization [Gast et al. 2015]) problem to solve for the discrete momentum balance. We solve these systems using Newton's method. However, the rotational invariance of the potential energy gives rise to discrete forces with indefinite Hessians. Given the similarity to hyperelasticity, we define a novel definiteness fix as in [Kim et al. 2019; Smith et al. 2019; Teran et al. 2005] to the surface tension force Hessian that allows for application of the conjugate gradient method in solving the linearized system at each Newton iteration. We summarize our contributions as

- An updated Lagrangian discretization of surface tension forces defined as the gradient of the surface energy with respect to the flow over a time step.

- A particle/level set based boundary particle quadrature rule for computing the surface area of a collection of discrete particles.

- Expressions for the eigenvalues and eigenvectors of the boundary quadrature surface tension energy Hessian which is used for a definiteness fix procedure as in [Teran et al. 2005].

\section{RELATED WORK}

We briefly discuss existing work related to the simulation of liquids with surface tension effects. We roughly divide discussion in terms of methods that use a Lagrangian mesh, those that use semi-implicit time stepping, and those that use particle-based discretization.

Surface tracking and Lagrangian meshes. Surface tracking techniques that resolve the liquid surface with explicit mesh topology have many advantages for surface tension effects since they accurately allow for curvature estimation and boundary application [Da et al. 2016; Müller 2009; Wojtan et al. 2010]. Brochu et al. [2010] use the surface tracking formulation of Brochu and Bridson [2009] to explicitly track topological changes of the liquid interface. This allows them to accurately resolve surface tension boundary conditions with a cut-cell Voronoi discretization based on the embedded tetrahedron formulation of Batty et al. [2010]. Sin et al. [2009] and De Goes et al. [2015] use similar Voronoi-based discretizations. While the previously mentioned techniques use an accompanying volumetric, usually Eulerian, discretization, Da et al. [2016] use a boundary element formulation with fluid represented by a tracked triangulation of its boundary. They use the surface tracking formulation of Da et al. [2014] to resolve merging and pinching behaviors. Thürey et al. [2010] also use a surface tracking approach and build on the formulation of Sussman and Ohta [2009] and implicit mean curvature flow approach of Eckstein et al. [2007]. Misztal et al. [2013] also define the surface tension force as the gradient of the surface area. They build on [Misztal and Bærentzen 2012] to develop an implicit approach for tetrahedron-based incompressible flows with surface tension. Wicke 
et al. [2010] use dynamic topology tetrahedron meshes. Zhu et al. [2014] use dynamic codimensional simplicial meshes to resolve thin sheets, filaments and droplets. They use the surface tension force approach of Zheng et al. [2015] combined with a novel rim-based surface tension on the boundary of thin sheets.

Particle based methods. PIC based approaches to fluid flow simulation are well established in the graphics community [Ando et al. 2013; Bridson 2008; Mercier et al. 2015]. Brackbill et al. [1992] developed the Continuum Surface Force (CSF) approach for PIC which defines normals and curvatures as gradients of color functions (defined on the grid after transferring from particles). The surface tension force is effectively regularized as color function transitions over a few cell widths. Müller [2003] et al. also use the gradient of a color function in a per-particle manner based on the work of Morris [2000], which is a generalization of the CSF model of Brackbill et al. [1992] to SPH. CSF has a number of drawbacks, including that: normalization of the color gradient is noisy for internal particles, curvature estimation is very sensitive to particle sampling uniformity and CSF forces are not exactly conservative. [Akinci et al. 2013; Becker and Teschner 2007; Clavet et al. 2005]. Yu et al. [2012] use surface tracking with SPH to define surface tension forces. In general, the determination of which particles should be considered to be on the boundary of a particle-based domain is an open problem [Dilts 2000; Haque and Dilts 2007; He et al. 2012; Sandim et al. 2016; Zhang et al. 2008; Zorilla et al. 2020]. Orthmann et al. [2013] use a discrete delta function approach with SPH to define particle-based surface area. Müller et al. combine SPH with surface tracking [Müller 2009]. Lastly, although our approach is the first fully implicit MPM discretization of surface tension, there are many existing fully implicit MPM discretizations of elastoplastic materials [Fei et al. 2018; Stomakhin et al. 2013; Wang et al. 2020].

Implicit Surface Tension. Popinet [2018] provides a useful review of implicit surface tension techniques. Bänsch et al. [2001] use a semi-implicit approach akin to one step of Newton iteration with an explicit mesh, FEM discretization of a surface energy-based formulation. Hysing [2006] uses a semi-implicit approach based on a variational CSF. Sussman and Ohta [2009] also use a semi-implicit approach based on mean curvature flow. Although these approaches are not fully implicit, Popinet [2018] shows they are equivalent to the addition of a surface viscosity that damps capillary waves and leads to an $O(\Delta x)$ time step. Hochstein and Williams [1996] developed one of the first implicit approaches for surface tension between two phases. Hou et al. [1994] add and subtract a Laplacian term as an approximation to the surface tension Hessian in a boundary integral formulation. Jarauta et al. [2018] use a Lagrangian formulation of incompressible flow and treat surface tension in a fully implicit manner. Zheng et al. [2015] develop a hybrid particle/grid based implicit technique for surface tension with a three-dimensional version of Schroeder et al. [2012] using a triangle mesh to discretize the surface tension force.

\section{FLOW KINEMATICS}

We use a continuum assumption [Gonzalez and Stuart 2008] and associate the spatial location of material at time $t$ with subsets

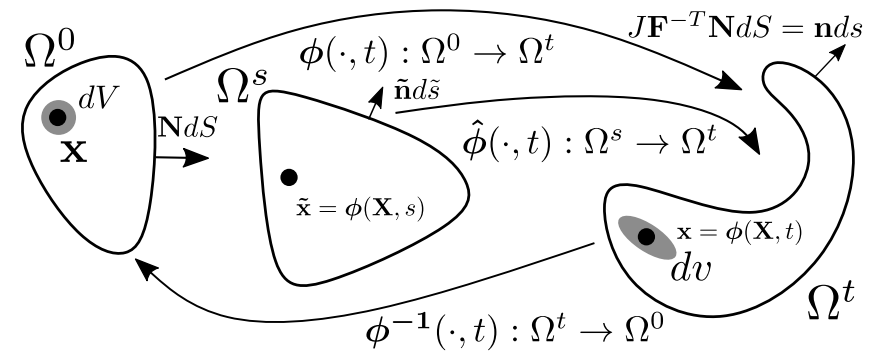

Fig. 2. We use an updated Lagrangian view of the flow map with either $\Omega^{0}$ or $\Omega^{s}$ used as a reference configuration. We demonstrate our notation for the kinematic flow quantities used in this formulation.

$\Omega^{t} \subset \mathbb{R}^{d}, d=2$, 3. Material motion is described by a flow map $\phi: \Omega^{0} \times[0, T] \rightarrow \mathbb{R}^{d}$ that defines trajectories of continuum particles of material $\left(\mathrm{X} \in \Omega^{0}\right)$ to their time $t$ locations $\left(\mathbf{x} \in \Omega^{t}\right)$ as $\phi(X, t)=$ $\mathrm{x}$. Here we use $\Omega^{t}=\left\{\mathrm{x} \in \mathbb{R}^{d} \mid \exists \mathrm{X} \in \Omega^{0}\right.$ such that $\left.\mathrm{x}=\phi(\mathrm{X}, t)\right\}$ to denote the time $t$ configuration of the material, where $\Omega^{0}=\{\mathrm{X}\}$ is the initial configuration of the material (see Figure 2).

Material velocity is obtained by differentiation in time $\mathbf{V}=\frac{\partial \phi}{\partial t}$. Differentiation in space defines the deformation gradient $F=\frac{\partial \phi}{\partial X}$ which captures the degree of non-rigidity of the motion. For example, rigid body motion is characterized by a spatially constant, orthogonal matrix $\mathbf{F}$. We use $J=\operatorname{det}(\mathbf{F})$ to denote the deformation gradient determinant. Intuitively, $J$ is the ratio of an initial infinitesimal volume at time $t(d v)$ to its preimage under the flow $(d V)$ in the initial configuration (see Figure 2). For example, incompressible flow is characterized by motion with $J=1$.

\subsection{Eulerian and updated Lagrangian Kinematics}

Quantities like $\phi, J$ and $\mathrm{F}$ defined over the reference configuration $\Omega^{0}$ are typically referred to as Lagrangian. However, since the configurations $\Omega^{0}$ and $\Omega^{t}$ are equivalent under the flow, we can write quantities over $\Omega^{t}$ as well. This change of variables defines what are typically referred to as Eulerian quantities. For example, the Eulerian velocity is related to the Lagrangian velocity through $\mathbf{v}(\phi(X, t), t)=\mathbf{V}(\mathrm{X}, t)$ and $\frac{\partial \mathbf{v}}{\partial t}(\phi(\mathrm{X}, t), t)+\frac{\partial \mathbf{v}}{\partial \mathbf{x}}(\phi(\mathrm{X}, t), t) \frac{\partial \phi}{\partial t}(\mathrm{X}, t)=$ $\frac{\partial \mathrm{V}}{\partial t}(\mathrm{X}, t)$. This can also be done with the inverse flow map $\phi^{-1}$ as $\mathbf{v}(\mathbf{x}, t)=\mathbf{V}\left(\phi^{-1}(\mathbf{x}, t), t\right)$ [Gonzalez and Stuart 2008]. It is this relation that motivates the definition of the material derivative $\frac{D \mathbf{v}}{D t}(\mathbf{x}, t)=\frac{\partial \mathrm{V}}{\partial t}\left(\phi^{-1}(\mathbf{x}, t), t\right)$. Some quantities like mass density $\rho$ are most commonly defined as Eulerian quantities. We refer to the Lagrangian counterpart of the mass density as $R: \Omega^{0} \times[0, T] \rightarrow \mathbb{R}$ with $R(\mathbf{X}, t)=\rho(\phi(\mathbf{X}, t), t)$.

Another variation of the Lagrangian view is updated Lagrangian [Belytschko et al. 2013]. In this case, quantities are written over an intermediate configuration $\Omega^{s}$ with $s<t$ (see Figure 2). For example, the velocity can be written as $\hat{\mathbf{v}}(\tilde{\mathbf{x}}, t)=\mathbf{V}\left(\phi^{-1}(\tilde{\mathbf{x}}, s), t\right)$ for $\tilde{\mathbf{x}} \in \Omega^{s}$. This quantity is essentially Lagrangian; for example, its time derivative does not require a material derivative $\frac{\partial \hat{\mathrm{v}}}{\partial t}(\tilde{\mathbf{x}}, t)=$ $\frac{\partial \mathrm{V}}{\partial t}\left(\boldsymbol{\phi}^{-1}(\tilde{\mathbf{x}}, s), t\right)$ since the term $\boldsymbol{\phi}^{-1}(\tilde{\mathbf{x}}, s)$ does not vary with time, as in the Eulerian case. We build our temporal discretization from the updated Lagrangian view. We will generally use upper case to 
denote Lagrangian quantities, hat superscript (with lower case) to denote updated Lagrangian quantities, and lower case for Eulerian quantities, e.g. $\mathbf{V} \Longleftrightarrow \hat{\mathbf{v}} \Longleftrightarrow \mathbf{v}$. We will make considerable use of the updated Lagrangian flow map $\left.\hat{\phi}(\tilde{\mathbf{x}}, t)=\phi\left(\phi^{-1}(\tilde{\mathbf{x}}, s), t\right)\right)$. Intuitively, this is the mapping from the time $s$ configuration $\Omega^{s}$ to the time $t$ configuration $\Omega^{t}$ under the flow.

\section{CONSERVATION OF MASS AND MOMENTUM}

The governing equations for the physical system are obtained from conservation of mass and momentum. These are most commonly written in Eulerian form as

$$
\rho \frac{D \mathbf{v}}{D t}=-\nabla p+\rho \mathbf{g}, \frac{D \rho}{D t}=-\rho \nabla \cdot \mathbf{v}, \mathbf{x} \in \Omega^{t} .
$$

with boundary conditions defined from surface tension $p=k^{\sigma} \kappa, \mathbf{x} \in$ $\partial \Omega_{N}^{t}$ and/or prescribed velocity $\mathbf{v} \cdot \mathbf{n}=v_{\mathrm{bc}}^{n}, \mathbf{x} \in \partial \Omega_{D}^{t}$ (see Figure 2 ). Here $\kappa$ is the mean curvature and $k^{\sigma}$ the coefficient of surface tension [Adamson and Gast 1967]. Furthermore, $p$ is the pressure in the fluid and $\mathrm{g}$ is the gravitational acceleration.

In order to penalize compressibility of the liquid, we use the constitutive relation $p=-k^{p}(j-1)$ where $k^{p}$ is the bulk modulus and $j(\mathbf{x}, t)=J\left(\phi^{-1}(\mathbf{x}, t), t\right)$ is the Eulerian deformation gradient determinant. Intuitively, larger values of the bulk modulus penalize compressible flow $(J \neq 1)$ more severely.

\subsection{Variational form and potential energy}

Our discretization of the momentum balance in Equation (1) is based on the potential energy of the material. The potential energy associated with surface tension is proportionate to the surface area of the material as it evolves in the flow [Adamson and Gast 1967]. We use $\Psi^{s}(\phi(\cdot, t))$ to denote the total surface tension potential energy at time $t$ under the flow map

$$
\Psi^{s}(\phi(\cdot, t))=k^{\sigma} \int_{\partial \Omega^{t}} d s(\mathbf{x})=k^{\sigma} \int_{\partial \Omega^{s}}\left|\hat{J}^{-T} \tilde{\mathbf{F}^{-T}}\right| d s(\tilde{\mathbf{x}}) .
$$

Here $k^{\sigma}$ is the coefficient of surface tension. Increasing values of the surface tension coefficient correspond to materials with large surface energies like water drops at small scales or liquid metals. We note that in Equation (2) the integral over $\partial \Omega^{t}$ can be written as one over $\partial \Omega^{s}$ using the surface integral change of variables, where $\tilde{\mathbf{n}}$ is the outward unit normal to the initial material boundary (see Figure 2). Here $\left|\hat{J} \hat{\mathbf{F}}^{-T} \tilde{\mathbf{n}}\right|$ can be shown to be the ratio of infinitesimal surface areas in the current $(d s)$ and time $s(d \tilde{s})$ configurations. The pressure and gravitational forces in the liquid can also be defined from their associated potential energies

$$
\Psi^{p}(\phi(\cdot, t))=\int_{\Omega^{0}} \frac{k^{p}}{2}(J-1)^{2} d \mathbf{X}, \Psi^{g}(\phi(\cdot, t))=\int_{\Omega^{0}} \phi \cdot R J \mathbf{g} d \mathbf{X} .
$$

For the pressure and surface tension potential energies, we also define the following potential energy densities

$$
\hat{\Psi}^{p}(J)=\frac{k^{p}}{2}(J-1)^{2}, \quad \hat{\Psi}^{s}(\hat{\mathbf{F}}, d \mathbf{A})=k^{\sigma}\left|\hat{J} \hat{\mathbf{F}}^{-T} d \mathbf{A}\right| .
$$

The momentum balance in Equation (1), including the surface tension and velocity boundary conditions, is equivalent to the variational form

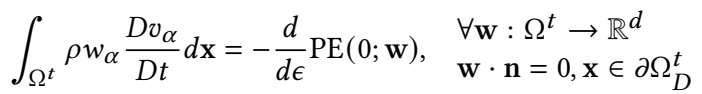

where $\operatorname{PE}(\epsilon ; \mathbf{w})=\Psi^{s}(\hat{\boldsymbol{\phi}}(\cdot, t)+\epsilon \hat{\mathbf{w}})+\Psi^{p}(\phi(\cdot, t)+\epsilon \mathbf{W})+\Psi^{g}(\phi(\cdot, t)+$ $\epsilon \mathbf{W})$ for $\hat{\mathbf{w}}(\tilde{\mathbf{x}})=\mathbf{w}(\hat{\boldsymbol{\phi}}(\tilde{\mathbf{x}}, t))$ and $\mathbf{W}(\mathbf{X})=\mathbf{w}(\phi(\mathbf{X}, t))$. This is obtained by taking the dot product of the momentum balance with $w_{\alpha}$ and integrating over $\Omega^{t}$, as well as integration by parts. The left hand side of Equation (4) can be written in the updated Lagrangian view by changing variables to $\Omega^{s}$ with $s<t$ resulting in

$$
\int_{\Omega^{t}} \rho w_{\alpha} \frac{D v_{\alpha}}{D t} d \mathbf{x}=\int_{\Omega^{s}} \hat{\rho} \hat{w}_{\alpha} \frac{\partial \hat{v}_{\alpha}}{\partial t} \hat{J} d \tilde{\mathbf{x}}
$$

where $\hat{\rho}(\tilde{\mathbf{x}}, t)=\rho(\hat{\boldsymbol{\phi}}(\tilde{\mathbf{x}}, t), t)$.

\section{DISCRETIZATION}

We approximate the governing equations with MPM and APIC [Jiang et al. 2015, 2016; Sulsky et al. 1994]. To discretize in space at time $t^{n}$ we use particle samples $\mathrm{x}_{p}^{n}$ of the domain $\Omega^{t^{n}}$. Each particle also approximates the deformation gradient determinant $J_{p}^{n}$ and velocity near the particle in terms of constant $\mathbf{v}_{p}^{n}$ and affine velocity $\mathbf{A}_{p}^{n}$. The initial mass $m_{p}=\rho\left(\mathbf{x}_{p}^{0}, 0\right) V_{p}^{0}$ and volume $V_{p}^{0}$ are also stored. Note that mass does not change with time since it is conserved and the time $t^{n}$ volume of the particle can be inferred from the deformation gradient determinant as $V_{p}^{n}=J_{p}^{n} V_{p}^{0}$. We extend traditional MPM by adding massless particles $\mathbf{x}_{q}^{n}$ with boundary area samples $d \mathrm{~A}_{q}^{n}$ for surface tension energy quadrature (see Figure 3 and Section 5.1). These samples are added at the beginning of each time step and removed at the end. By their massless nature, this resampling does not affect conservation of mass and momentum.

We first transfer the time $t^{n}$ particle mass and momentum to the grid using APIC transfers [Jiang et al. 2015] with quadratic B-spline interpolating functions $N_{\mathbf{i}}(\mathbf{x})=N\left(\mathbf{x}-\mathbf{x}_{\mathbf{i}}\right)$ defined on the Eulerian grid nodes $\mathbf{x}_{\mathbf{i}}$ as

$m_{\mathbf{i}}^{n}=\sum_{p} m_{p} N_{\mathbf{i}}\left(\mathbf{x}_{p}^{n}\right), \quad m_{\mathbf{i}}^{n} \mathbf{v}_{\mathbf{i}}^{n}=\sum_{p} m_{p} N_{\mathbf{i}}\left(\mathbf{x}_{p}^{n}\right)\left(\mathbf{v}_{p}^{n}+\mathbf{A}_{p}^{n}\left(\mathbf{x}_{\mathbf{i}}-\mathbf{x}_{p}^{n}\right)\right)$.

Note that we do not sum over the surface particles since they have no mass. Next we update grid momentum by discretizing the right hand side of Equation (5) with $s=t^{n}$ as

$$
\int_{\Omega^{s}} \hat{\rho} \hat{w}_{\alpha} \frac{\partial \hat{v}_{\alpha}}{\partial t} \hat{J} d \tilde{\mathbf{x}} \approx \int_{\Omega^{t^{n}}} \hat{\rho} \hat{w}_{\alpha} \frac{\hat{v}_{\alpha}\left(\tilde{\mathbf{x}}, t^{n+1}\right)-v_{\alpha}\left(\tilde{\mathbf{x}}, t^{n}\right)}{\Delta t} \hat{J} d \tilde{\mathbf{x}} .
$$

Here we use a backward difference in time. To discretize in space, we approximate $v_{\alpha}, \hat{v}_{\alpha}$ and $\hat{w}_{\alpha}$ using the quadratic B-splines as $v_{\alpha}\left(\tilde{\mathbf{x}}, t^{n}\right)=v_{\mathbf{i} \alpha}^{n} N_{\mathbf{i}}(\tilde{\mathbf{x}}), \hat{v}_{\alpha}\left(\tilde{\mathbf{x}}, t^{n+1}\right)=\hat{v}_{\mathbf{i} \alpha}^{n+1} N_{\mathbf{i}}(\tilde{\mathbf{x}})$ and $\hat{w}_{\alpha}=\delta_{\beta \alpha} N_{\mathbf{i}}(\tilde{\mathbf{x}})$ respectively. The integral (7) then becomes

$$
\left(\int_{\Omega^{t^{n}}} \hat{\rho} \hat{J} N_{\mathbf{i}} N_{\mathbf{j}} d \tilde{\mathbf{x}}\right) \frac{\hat{v}_{\mathbf{i} \beta}^{n+1}-v_{\mathbf{i} \beta}^{n}}{\Delta t} .
$$


We approximate the integral using the MPM particles as quadrature points:

$$
\begin{aligned}
\int_{\Omega^{t^{n}}} \hat{\rho} \hat{J} N_{\mathbf{i}} N_{\mathbf{j}} d \tilde{\mathbf{x}} & \approx \sum_{p} \hat{\rho}\left(\mathbf{x}_{p}^{n}, t^{n+1}\right) \hat{J}\left(\mathbf{x}_{p}^{n}, t^{n+1}\right) V_{p}^{n} N_{\mathbf{i}}\left(\mathbf{x}_{p}^{n}\right) N_{\mathbf{j}}\left(\mathbf{x}_{p}^{n}\right) \\
& =\sum_{p} R\left(\mathbf{x}_{p}, t^{n+1}\right) J\left(\mathbf{x}_{p}, t^{n+1}\right) V_{p}^{0} N_{\mathbf{i}}\left(\mathbf{x}_{p}^{n}\right) N_{\mathbf{j}}\left(\mathbf{x}_{p}^{n}\right) \\
& =\sum_{p} R\left(\mathbf{x}_{p}, t^{0}\right) V_{p}^{0} N_{\mathbf{i}}\left(\mathbf{x}_{p}^{n}\right) N_{\mathbf{j}}\left(\mathbf{x}_{p}^{n}\right) \\
& =\sum_{p} m_{p} N_{\mathbf{i}}\left(\mathbf{x}_{p}^{n}\right) N_{\mathbf{j}}\left(\mathbf{x}_{p}^{n}\right)
\end{aligned}
$$

where $R$ is the Lagrangian mass density defined by $R\left(\mathrm{X}_{p}, t\right)=$ $\rho\left(\phi\left(\mathbf{X}_{p}, t\right), t\right)$, and $\mathbf{X}_{p}=\phi^{-1}\left(\mathbf{x}_{p}^{n}, t^{n}\right)=\mathbf{x}_{p}^{0}$. If we replace this integral with the lumped mass approximation by summing on the index $\mathbf{j}$, we obtain the mass transfer in (6).

In order to update the grid momentum (and thus compute $\hat{v}_{\mathbf{i} \beta}^{n+1}$ ) we first discretize the total potential energy using the MPM points for quadrature:

$$
e(\hat{\mathbf{x}})=\sum_{p} \hat{\Psi}^{p}\left(J_{p}^{n+1}(\hat{\mathbf{x}})\right) V_{p}^{n}+\sum_{q} \hat{\Psi}^{s}\left(\hat{\mathbf{F}}_{q}(\hat{\mathbf{x}}), d \mathbf{A}_{q}\right) \approx \operatorname{PE}(0 ; \mathbf{w}) .
$$

Note that the massless surface tension particles are used in this discretization. We use $\hat{\mathbf{x}}$ to denote the vector of all potentially moved grid node positions $\hat{\mathbf{x}}_{\mathbf{i}}$ where the functions $J_{p}^{n+1}(\hat{\mathbf{x}}), \hat{F}_{q}(\hat{\mathbf{x}})$ are given by

$$
J_{p}^{n+1}(\hat{\mathbf{x}})=\left(1+\left(\hat{\mathbf{x}}_{\mathbf{i}}-\mathbf{x}_{\mathbf{i}}\right) \cdot \frac{\partial N_{\mathbf{i}}}{\partial \mathbf{x}}\left(\mathbf{x}_{p}^{n}\right)\right) J_{p}^{n}, \quad \hat{\mathbf{F}}_{q}(\hat{\mathbf{x}})=\hat{\mathbf{x}}_{\mathbf{i}} \frac{\partial N_{\mathbf{i}}^{T}}{\partial \mathbf{x}}\left(\mathbf{x}_{q}^{n}\right) .
$$

The force on grid node $\mathbf{i}$ is then $\mathbf{f}_{\mathbf{i}}(\hat{\mathbf{x}})=-\frac{\partial e}{\partial \hat{\mathbf{x}}_{\mathbf{i}}}(\hat{\mathbf{x}})$ and the update for the grid momentum is then

$$
m_{\mathbf{i}}^{n} \frac{\hat{\mathbf{v}}_{\mathbf{i}}^{n+1}-\mathbf{v}_{\mathbf{i}}^{n}}{\Delta t}=\mathbf{f}_{\mathbf{i}}(\mathbf{x}+\Delta t \hat{\mathbf{q}})+m_{\mathbf{i}}^{n} \mathbf{g}
$$

where $\hat{\mathbf{q}}=\mathbf{0}$ for explicit time integration and $\hat{\mathbf{q}}=\hat{\mathbf{v}}^{n+1}$ for backward Euler time integration. As with $\hat{\mathbf{x}}$, the vector $\hat{\mathbf{v}}^{n+1}$ contains the corresponding velocity vectors defined on each grid node. Furthermore, $\mathbf{x}$ is the vector of all unmoved grid node locations $\mathbf{x}_{\mathbf{i}}$. The $\alpha$ component of the force on grid node $\mathbf{i}$ is then

$$
\begin{aligned}
f_{\mathbf{i} \alpha}(\hat{\mathbf{x}})= & -\sum_{p} \frac{\partial \hat{\Psi}^{p}}{\partial J}\left(J_{p}^{n+1}(\hat{\mathbf{x}})\right) \frac{\partial N_{\mathbf{i}}}{\partial x_{\alpha}}\left(\mathbf{x}_{p}^{n}\right) J_{p}^{n} V_{0}^{n} \\
& -\sum_{q} \frac{\partial \hat{\Psi}^{s}}{\partial \hat{F}_{\alpha \gamma}}\left(\hat{\mathbf{F}}_{q}(\hat{\mathbf{x}}), d \mathbf{A}_{q}\right) \frac{\partial N_{\mathbf{i}}}{\partial x_{\gamma}}\left(\mathbf{x}_{q}^{n}\right) .
\end{aligned}
$$

After the grid update, we use APIC to transfer from grid to particles

$$
\mathbf{v}_{p}^{n+1}=\sum_{\mathbf{i}} N_{\mathbf{i}}\left(\mathbf{x}_{p}^{n}\right) \hat{\mathbf{v}}_{\mathbf{i}}^{n+1}, \quad \mathbf{A}_{p}^{n+1}=\frac{4}{\Delta x^{2}} \sum_{\mathbf{i}} N_{\mathbf{i}}\left(\mathbf{x}_{p}^{n}\right) \hat{\mathbf{v}}_{\mathbf{i}}^{n+1}\left(\mathbf{x}_{\mathbf{i}}-\mathbf{x}_{p}^{n}\right)^{T}
$$

The particles are then updated to their time $t^{n+1}$ positions via $\mathrm{x}_{p}^{n+1}=\mathrm{x}_{p}^{n}+\Delta t \mathrm{v}_{p}^{n+1}$. The MPM discretization using APIC transfers is summarized in Algorithm 1.
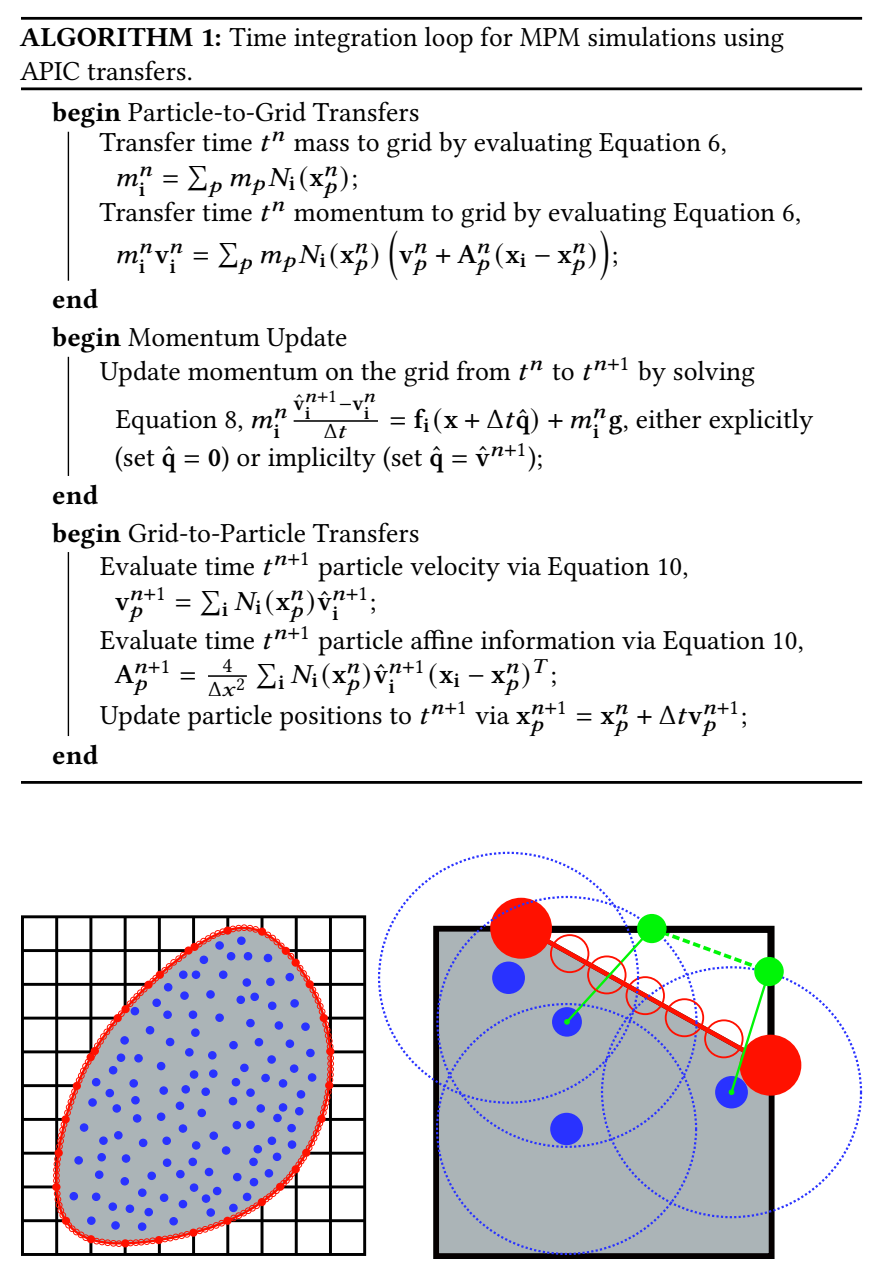

Fig. 3. (Left) MPM fluid particles (dark blue) give rise to a level set isocontour (red). (Right) The isocontour is of the level set formed by the union of each fluid particle's spherical level set. Surface tension forces are evaluated at sample points (open red circles) placed on the isocontour face.

\subsection{Surface Tension Boundary Sampling}

Here we describe our approach for seeding the surface tension quadrature particles $\mathbf{x}_{q}^{n}$ with area weighted normals $d \mathbf{A}_{q}$ on $\partial \Omega^{t^{n}}$. As is common in particle-based methods (e.g. [Boyd and Bridson 2012]), we define a spherical level set around each fluid particle and union the individual level sets to form an implicit representation $\varphi$ of $\Omega^{t^{n}}$. We then define $\partial \Omega^{t^{n}}$ as the zero isocontour of $\varphi$. However, the isocontour may be relatively distant from the fluid particles due to the level set radii of the particles (typically set as approximately $.4 \Delta x$ ), which can lead to e.g. particle distribution artifacts. We uniformly shift $\varphi$ by a multiple of $\Delta x$ (typically we choose $.2 \Delta x$ ) before computing the isocontour to account for this (see Figure 3). Additionally, we found that performing one iteration of Laplace smoothing on $\varphi$ resulted in smoother isocontours which is more desirable for surface tension forces. Simply adding a shift to the level set does this as well, but we found that only shifting led to a more jagged zero isocontour 


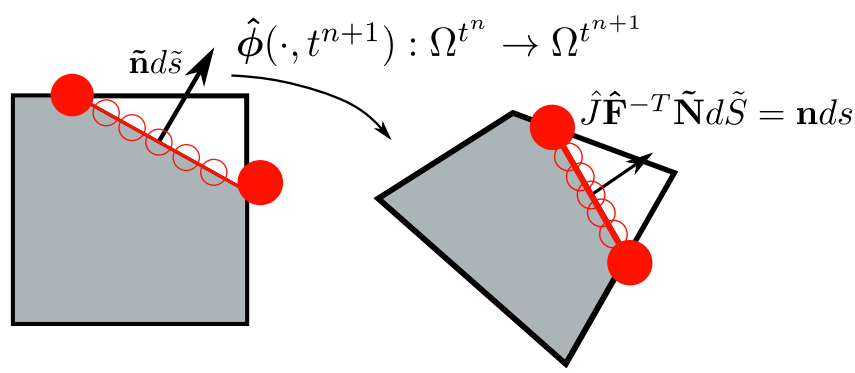

Fig. 4. (Left) The isocontour face normal with each sampled point being provided a surface area-weighted normal. (Right) The isocontour face normals and areas are updated with the mapping between $t^{n}$ and $t^{n+1}$.

and that a combination of both shifting and smoothing gave better results in practice.

After creating the isocontours, we sample $\mathrm{x}_{q}^{n}$ and $d \mathrm{~A}_{q}$ on each face (see Figure 3). In two dimensions, we sample equally-spaced points along each face, using a number of points that yield approximately constant distances between sample points across the entire isocontour. In three dimensions, we select face sample points using random barycentric coordinates. We choose the number of points sampled per face in order to achieve approximately evenly distributed sample points over $\partial \Omega^{t^{n}}$. In both two and three dimensions, we assign the surface area-weighted normal $d \mathbf{A}_{q}$ such that the surface area of an isocontour face is evenly distributed among sample points on that face and the direction is the isocontour face normal (see Figure 4). Figure 5 shows that this novel quadrature rule is at least first-order accurate under spatial refinement for the case of a $2 \mathrm{D}$ disc of radius $0.2 \mathrm{~m}$ filled with 64 random particles per grid cell and 10 boundary particles sampled per isocontour face.

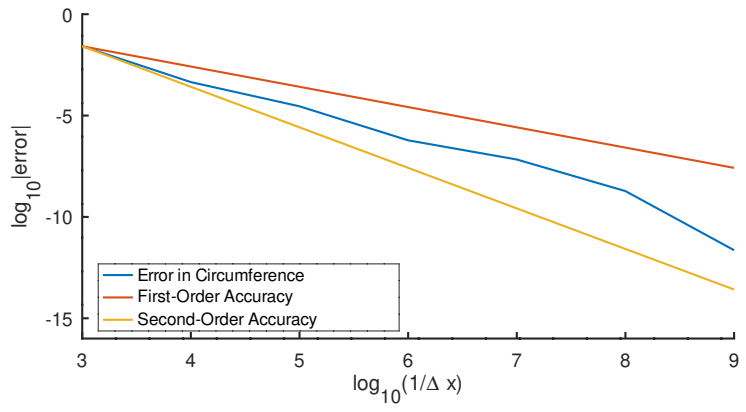

Fig. 5. Convergence of the total estimated surface area $\sum_{q}\left|d \mathrm{~A}_{q}\right|$ as the background grid is refined for a 2D disc.

\subsection{Fully-Implicit Formulation}

In the implicit case, summarized in Algorithm 2, we solve Equation (8) with $\hat{\mathbf{q}}=\hat{\mathbf{v}}^{n+1}$,

$$
m_{\mathrm{i}}^{n} \frac{\hat{v}_{\mathbf{i} \alpha}^{n+1}-v_{\mathbf{i} \alpha}^{n}}{\Delta t}=f_{\mathbf{i} \alpha}\left(\mathbf{x}+\Delta t \hat{\mathbf{v}}^{n+1}\right)+m_{\mathbf{i}}^{n} g_{\alpha}
$$

ACM Trans. Graph., Vol. 39, No. 6, Article 183. Publication date: December 2020. for $\hat{\mathbf{v}}^{n+1}$ using Newton's method. We solve the resulting system using conjugate gradient. This requires the derivative of the force

$$
\begin{aligned}
\frac{\partial f_{\mathbf{i} \alpha}}{\partial \hat{x}_{\mathbf{j} \beta}}(\hat{\mathbf{x}})= & -\sum_{p} \frac{\partial^{2} \hat{\Psi}^{p}}{\partial J^{2}}\left(J_{p}^{n}(\hat{\mathbf{x}})\right) J_{p}^{n} \frac{\partial N_{\mathbf{i}}}{\partial x_{\alpha}}\left(\mathbf{x}_{p}^{n}\right) J_{p}^{n} \frac{\partial N_{\mathbf{j}}}{\partial x_{\beta}}\left(\mathbf{x}_{p}^{n}\right) V_{p}^{n} \\
& -\sum_{q} \frac{\partial^{2} \hat{\Psi}^{s}}{\partial \hat{F}_{\beta \delta} \partial \hat{F}_{\alpha \gamma}}\left(\hat{\mathbf{F}}_{q}^{n}(\hat{\mathbf{x}}), d \mathbf{A}_{q}\right) \frac{\partial N_{\mathbf{i}}}{x_{\gamma}}\left(\mathbf{x}_{q}^{n}\right) \frac{\partial N_{\mathbf{j}}}{x_{\delta}}\left(\mathbf{x}_{q}^{n}\right) .
\end{aligned}
$$

The Hessian $\partial^{2} \hat{\Psi}^{2} / \partial \hat{\mathbf{F}}^{2}$ of the surface tension energy density is indefinite in three dimensions. However, we can analytically determine its eigenstructure as in Table 1 . In the table, $\mathbf{b}_{1}$ and $\mathbf{b}_{2}$ are any unit

\begin{tabular}{|c|c|}
\hline Eigenvalue & Eigenvectors \\
\hline$k^{\sigma}|d \mathbf{A}|$ & $\begin{array}{l}\frac{1}{\sqrt{2}} \mathbf{b}_{1} \otimes \mathbf{b}_{2}+\frac{1}{\sqrt{2}}\left(\frac{\hat{J} \hat{\mathbf{F}}^{-T}}{\left|\hat{J} \hat{\mathbf{F}}^{-T}\right|} \times \mathbf{b}_{1}\right) \otimes\left(\frac{d \mathbf{A}}{|d \mathbf{A}|} \times \mathbf{b}_{2}\right) \\
\frac{1}{\sqrt{2}} \mathbf{b}_{1} \otimes\left(\frac{d \mathbf{A}}{|d \mathbf{A}|} \times \mathbf{b}_{2}\right)-\frac{1}{\sqrt{2}}\left(\frac{\hat{J} \hat{\mathbf{F}}^{-T}}{\left|\hat{J} \hat{\mathbf{F}}^{-T}\right|} \times \mathbf{b}_{1}\right) \otimes \mathbf{b}_{2}\end{array}$ \\
\hline$-k^{\sigma}|d \mathbf{A}|$ & $\begin{array}{l}\frac{1}{\sqrt{2}} \mathbf{b}_{1} \otimes \mathbf{b}_{2}-\frac{1}{\sqrt{2}}\left(\frac{\hat{J} \hat{\mathbf{F}}^{-T}}{\left|\hat{\jmath} \hat{\mathbf{F}}^{-T}\right|} \times \mathbf{b}_{1}\right) \otimes\left(\frac{d \mathbf{A}}{|d \mathbf{A}|} \times \mathbf{b}_{2}\right) \\
\frac{1}{\sqrt{2}} \mathbf{b}_{1} \otimes\left(\frac{d \mathbf{A}}{|d \mathbf{A}|} \times \mathbf{b}_{2}\right)+\frac{1}{\sqrt{2}}\left(\frac{\hat{J} \hat{\mathbf{F}}^{-T}}{\left|\hat{\jmath} \hat{\mathbf{F}}^{-T}\right|} \times \mathbf{b}_{1}\right) \otimes \mathbf{b}_{2}\end{array}$ \\
\hline$k^{\sigma} \frac{|d \mathbf{A}|^{2}}{\left|\hat{J} \hat{\mathbf{F}}^{-T}\right|}\left|\hat{\mathbf{F}} \mathbf{w}_{1}\right|^{2}$ & $\frac{\hat{J} \hat{\mathbf{F}}^{-T}}{\left|\hat{J} \hat{\mathbf{F}}^{-T}\right|} \otimes \mathbf{w}_{0}$ \\
\hline$k^{\sigma} \frac{|d \mathbf{A}|^{2}}{\left|\hat{J} \hat{\mathbf{F}}^{-T}\right|}\left|\hat{\mathbf{F}} \mathbf{w}_{0}\right|^{2}$ & $\frac{\hat{J} \hat{\mathbf{F}}^{-T}}{\mid \hat{J} \hat{\mathbf{F}}^{-T \mid}} \otimes \mathbf{w}_{1}$ \\
\hline 0 & $\begin{array}{l}\mathbf{u}_{0} \otimes \frac{d \mathrm{~A}}{|d \mathrm{~A}|} \\
\mathbf{u}_{1} \otimes \frac{d \mathrm{~A}}{|d \mathrm{~A}|} \\
\mathbf{u}_{2} \otimes \frac{d \mathbf{A}}{|d \mathbf{A}|}\end{array}$ \\
\hline
\end{tabular}

Table 1. Complete eigenstructure of the Hessian of the surface tension energy density $\partial^{2} \hat{\Psi}^{2} / \partial \hat{\mathrm{F}}^{2}$.

vectors orthogonal to $d \mathbf{A}$ and $\hat{J} \hat{\mathbf{F}}^{-T}$ respectively, $\mathbf{w}_{0}$ and $\mathbf{w}_{1}$ are any orthonormal vectors orthogonal to $d \mathbf{A}$ satisfying $\hat{\mathbf{F}} \mathbf{w}_{0} \cdot \hat{\mathbf{F}} \mathbf{w}_{1}=0$, and $\mathbf{u}_{0}, \mathbf{u}_{1}$, and $\mathbf{u}_{2}$ are any orthonormal basis for $\mathbb{R}^{3}$. We refer readers to the supplementary material [Hyde et al. 2020] for a proof. Note that there is one negative eigenvalue with multiplicity two and a zero eigenvalue with multiplicity three. We perform a definiteness fix as in [Kim et al. 2019; Smith et al. 2019; Teran et al. 2005] by clamping these negative eigenvalues to 0 . The corresponding term in the Jacobian matrix for Newton's method will then be positive semi-definite.

\subsection{Hydrostatic Solution}

Unlike many Eulerian fluid simulations, it is non-trivial to obtain a hydrostatic solution for a fluid at rest when the fluid is represented with particles, as in MPM. In order to achieve the hydrostatic solution (to within machine precision), the following equation must be satisfied exactly:

$$
-\int_{\Omega} N_{\mathrm{i}} \frac{\partial p}{\partial x_{\alpha}} d \mathbf{x}+\int_{\Omega} \rho g_{\alpha} N_{\mathbf{i}} d \mathbf{x}=0,
$$




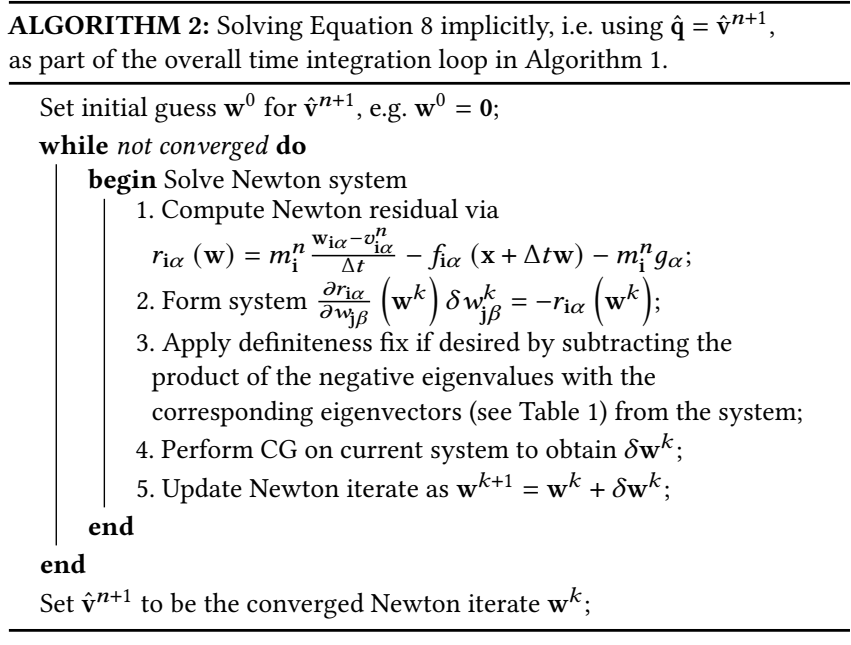

which arises from the balance of the internal and gravitational forces in the conservation of momentum (see [Hyde et al. 2020]). Given our expression for $\Psi^{p}$, we show that we can choose the initial particle locations and volumes as a combination of Gauss quadrature points/volumes and random points/volumes since the integrands are of order 4 in two dimensions and 6 in three dimensions. It suffices to use Gauss points alone; however this biases the simulation with unwanted and persistent particle structure.

We build on [Patterson et al. 2012] and determine our quadrature rule from the least squares approximation to the bi-quadratic/triquadratic integrals forming the linear system $\mathrm{C}=\mathrm{Aw}$ with $\mathrm{C}$ being the evaluated integrals of the monomial basis, $\mathrm{A}$ being the matrix of integrands/monomials evaluated at each particle position, and w being the unknown quadrature volumes. To obtain a positive solution to this system as required, we solve the following KKT system that minimizes $k\left(w_{i}-\frac{V_{c}}{N_{p}}\right)^{2}$ subject to the constraint $\mathrm{C}=\mathrm{Aw}$, where $V_{c}$ is the total volume of a grid cell, $N_{p}$ is the number of particles in a given cell, and $k>0$ is a scalar:

$$
\left[\begin{array}{cc}
k & \mathrm{~A}^{T} \\
\mathbf{A} & 0
\end{array}\right]\left[\begin{array}{c}
\mathbf{w} \\
\lambda
\end{array}\right]=\left[\begin{array}{c}
\frac{V_{c}}{N_{p}} \\
\mathbf{C}
\end{array}\right] .
$$

For more details on the derivation and solution of the KKT system, we refer readers to the supplementary material [Hyde et al. 2020].

To validate our treatment of the hydrostatic case, we ran a twodimensional simulation of a tank partially filled with static fluid for 1,000 time steps using the explicit formulation of MPM with a maximum $\Delta t$ of $1 \times 10^{-3} \mathrm{~s}$. We measured an $L_{\infty}$-norm error in fluid particle velocity of $2.04 \times 10^{-12}$ (near machine precision). With the same example under the implicit MPM formulation, no numerical error is incurred. This is because we use a zero initial guess for the updated velocity vector in the linear solves during the Newton iterations, which converges with no numerical operations since the initial guess is the solution; hence an exactly zero state is maintained throughout the simulation. The effect of our hydrostatic formulation is demonstrated in Figure 6, which shows a 3D standing pool after 1,000 time steps $\left(\Delta t=1 \times 10^{-3} \mathrm{~s}\right)$ with our hydrostatic initialization and with random initialization (both surfaces start flat).

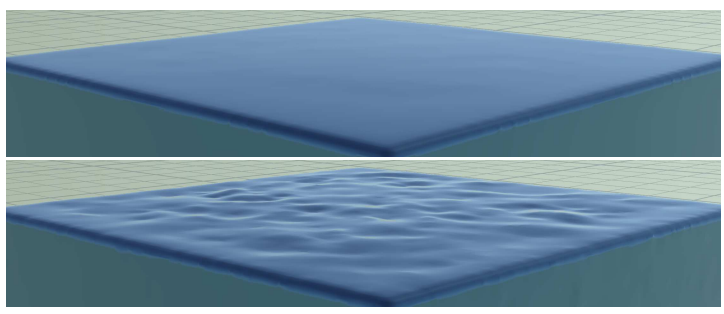

Fig. 6. A static 3D tank after 1,000 time steps with (top) and without (bottom) our hydrostatic initialization.

\subsection{Stability}

On a related note, we emphasize that spurious particle velocities or parasitic currents can develop in situations such as a stationary twodimensional circle of randomly-sampled particles in zero gravity. We simulate such a scenario with circle radius $0.1 \mathrm{~m}$, a large surface tension coefficient $k^{\sigma}=10.0$, grid resolution $\Delta x=1 / 31$, time step $\Delta t=1 \times 10^{-4} \mathrm{~s}$, and 16 particles per cell using implicit MPM; results are shown in Figure 7. The experiment also simulates a translating circle under similar conditions. We hypothesize that some of the small, oscillatory, but spurious currents that develop are due to the particles imperfectly sampling a circular geometry, though other factors such as $\Delta x$ and $\Delta t$ also have an influence; studying these effects is interesting future work

We also evaluate the stability of our method when run using explicit, semi-implicit (one Newton iteration), and implicit formulations of MPM. For this stability experiment, we simulate an oscillating 2D ellipse using various $\Delta x$ and various $\Delta t$, and assess whether each result is stable, unstable, or has significant artifacts; see Table 3. The distinction between stable and unstable can be unclear when using semi-implicit time stepping. Often these simulations do not technically go unstable, but instead exhibit unacceptable artifacts associated with failure to reduce the non-linear residual of the backward Euler system. We demonstrate these artifacts in Figure 8 where examples of stable and artifact-ridden results for implicit and semi-implicit time stepping are shown along with a comparison of plots of the backward Euler residual evaluated at each time step.

\subsection{One- and Two-Way Coupling}

Our modification of MPM to accommodate high surface energy fluids naturally allow use of existing techniques for one- and two-way coupling. One-way coupling can be achieved by specifying background grid nodes where boundary conditions such as zero normal velocity are defined, and then applying those boundary conditions between the particle-to-grid and grid-to-particle transfers. For twoway coupling between fluids and elastic meshes, we leverage the Lagrangian force framework of [Jiang et al. 2015].

\section{EXAMPLES}

We demonstrate the effectiveness and simplicity of our approach with a number of examples in two and three dimensions. Unless otherwise noted, our simulation domains are unit boxes, the bulk 


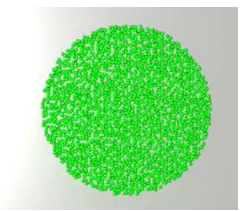

(a) $t=0 \mathrm{~s}$

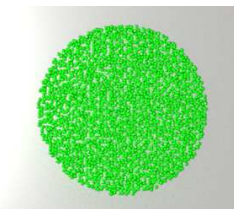

(d) $t=0 \mathrm{~s}$

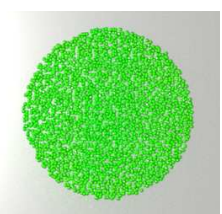

(b) $t=0.25 \mathrm{~s}$

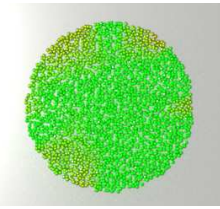

(e) $t=0.25 \mathrm{~s}$

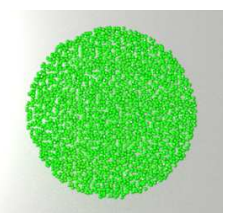

(c) $t=0.5 \mathrm{~s}$

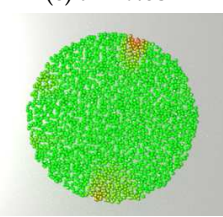

(f) $t=0.5 \mathrm{~s}$

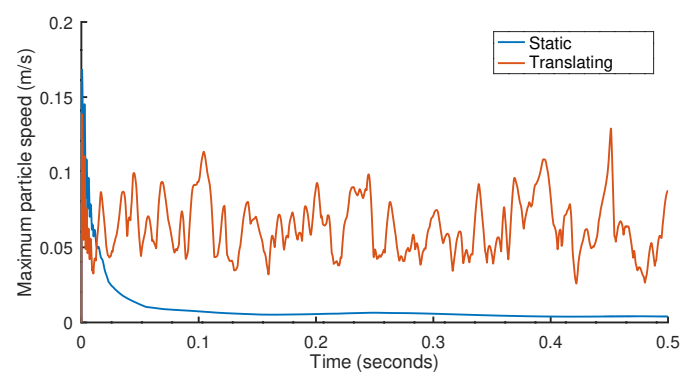

(g) Maximum relative velocities

Fig. 7. (Top) An initially stationary circle over time, with $\Delta t=1 \times 10^{-4} \mathrm{~s}$ and $k^{\sigma}=10.0$. Particles are colored by speed from $0 \mathrm{~m} / \mathrm{s}$ (green) to $0.1 \mathrm{~m} / \mathrm{s}$ (red). (Middle) A translating circle with initial velocity $(0.1,0.2) \mathrm{m} / \mathrm{s}$. (Bottom) Maximum relative speeds of particles in the static and translating circles.

modulus of the fluid is 416.67 , and the isocontour shift used when generating surface tension sample points is $0.2 \Delta x$.

Colliding Two-Dimensional Disks. Figure 9 demonstrates the effects of varying the surface tension coefficient $k^{\sigma}$ in the case of two two-dimensional disks of radius .125 colliding. The disks have initial velocity \pm 0.4 in all cases. The top row of figures, with the lowest surface tension $\left(k^{\sigma}=0.05\right)$, demonstrates smooth topological transitions as the disks pinch off and subsequently re-collide. As surface tension increases (middle and bottom rows, $k^{\sigma}=0.1,5.0$ ), the disks only merge once and oscillate with higher frequency. All cases use a background grid with $64^{2}$ cells and explicit MPM.

Qualitative Comparison with Experiment. To qualitatively assess physicality of our method under extremely high surface tension conditions, we produced a simulation (Figure 10) of a falling spherical droplet with $k^{\sigma}=20$ to match an experiment of a droplet impacting a superhydrophobic surface. The geometry and bouncing and recoiling behaviors of the droplet in our simulation, run using a background grid with $64^{3}$ cells and explicit MPM with a maximum $\Delta t$ of $1 \times 10^{-4} \mathrm{~s}$, closely match those observed in the experiment Moreover, our simulation produces smooth, stable results despite the significant stiffness of the problem.

Dam Break. While our novel focus is on liquids with large surface energy, we confirm that our methodology also applies to fluids with

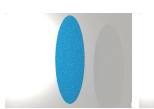

(a) $0 \mathrm{~s}$

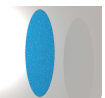

(g) $0 \mathrm{~s}$

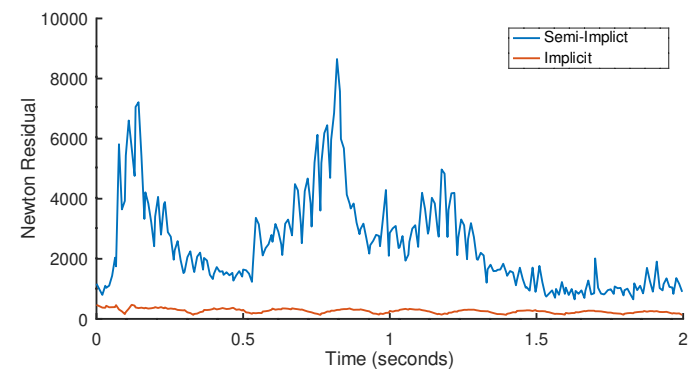

(m) Final Newton Residuals vs. Time
Fig. 8. An oscillating 2D ellipse with $k^{\sigma}=1000, \Delta t=0.01$, and $\Delta x=1 / 128$. (Top) Implicit MPM (5 Newton iterations, 5 CG iterations). (Middle) Semiimplicit (1 Newton iteration, 25 CG iterations). (Bottom) Semi-implicit MPM fails to obtain a low residual for the solves and hence is more susceptible to instability and artifacts.

low or moderate surface tension forces. Figure 11 shows a dam break flow in a cubic tank with varying surface tension coefficients, demonstrating significant turbulent splashing effects at low $k^{\sigma}$. Implicit MPM was used with a background grid with $64^{3}$ cells and a fixed $\Delta t$ of $2.5 \times 10^{-4} \mathrm{~s}$.

Moderate Surface Tension Droplet. Similar to the previous example, Figure 12 shows that our method can resolve interesting dynamic behavior at moderate surface tensions as in [Da et al. 2016]. A falling droplet was simulated with $k^{\sigma}=0.5$ using implicit MPM, a background grid with $128^{3}$ cells, and a fixed $\Delta t$ of $5 \times 10^{-3} \mathrm{~s}$. As noted in Table 2 , our simulation runs in about 5.98 s per timestep and hence about 41.8 s per frame on average. The analogous example in [Da et al. 2016] runs in a mean of $6.465 \mathrm{~s}$ and a maximum of $108.99 \mathrm{~s}$ per frame, though their simulation has a mean of only 1,898 vertices and 3,791 faces while our simulation has a comparatively large particle count of over 558k. In Figure 13 we demonstrate our approach with moderate surface energies in a comparison with the SPH approach of Akinci et al. [2013]. We adopt their example with a droplet falling into a pool causing a characteristic splash. Our method is able to achieve behaviors on par with their approach.

Relaxing Ellipsoid with Gravity. We simulated a liquid mercurylike ellipsoid which falls under gravity into a tank while also relaxing under surface tension effects. Figure 14 demonstrates this numerical experiment using several different values of $k^{\sigma}$, ranging from 0.025 to 1.0 , on a background grid with $64^{3}$ cells. Implicit MPM was used with a $\Delta t$ between $1 \times 10^{-3} \mathrm{~s}$ and $1 \times 10^{-2} \mathrm{~s}$. With lower surface tension coefficients, the material is highly dynamic as surface tension forces do not significantly influence the fluid's motion; however, 
sufficiently high surface tension coefficients allow the fluid to maintain its original topology as it deforms much less than with lower values of $k^{\sigma}$.

Oscillating Cube. As an example of a geometry relaxing under surface tension forces without gravity, we considered a cube of sidelength 0.2 with $k^{\sigma}=0.01$. Using implicit MPM with a fixed time step of $5 \times 10^{-3} \mathrm{~s}$ and a background grid with $128^{3}$ cells, Figure 15 demonstrates how the cube initially deforms into an octahedral configuration, before further oscillations which eventually lead to a spherical shape as numerical damping removes energy from the system. Particle renderings of the fluid and massless boundary sampling particles are also shown

Droplets on Platforms. As an example of one-way coupling with high surface energy liquids, we show a droplet with $k^{\sigma}=5.0$ falling through a series of platforms in Figure 16. A grid resolution of $64 \times 128 \times 64$ was used to accommodate a domain size of $1 \times 2 \times 1$. The droplet energetically recoils off the first platform and subsequently undergoes several topology changes as the droplet separates into two components which re-merge, eventually settling as a single component on the ground.

Droplet on Cloth Sheet. Finally, we present a two-way coupled example of a high surface energy liquid interacting with a thin Lagrangian cloth mesh in Figure 17. A resolution of $64^{3}$ was used for the background grid, while the cloth mesh was simulated with a resolution of $224 \times 4 \times 224$ mesh particles. Fluid is seeded using $665 \mathrm{~K}$ particles in a sphere of radius 0.15 . Grid nodes surrounding the corners of the cloth are set as fixed points to prevent velocity from being transferred to the corresponding mesh nodes. Implicit MPM was used with maximum and minimum $\Delta$ of $1 \times 10^{-3}$ and $1 \times 10^{-5}$ respectively. The bulk modulus of the fluid was set to $5,833.33$ while that of the mesh was set to $20,833.33$. $k^{\sigma}$ was chosen to be 1.0. Despite the stiffness of the mesh (which will result in rapid oscillations in the absence of fluid), the fluid weighs down the mesh and hinders its motion, while the cloth simultaneously induces motion in the fluid as it oscillates.

Surface Tension Performance. We report wall clock time for one time step of several of our examples in Table 2. Measurements were performed on a machine with an Intel Xeon Platinum 8275CL CPU running at $3.00 \mathrm{GHz}$. While our implementation could be improved (e.g. leveraging GPUs, additional parallelism, etc.), we emphasize that the cost of incorporating our surface tension formulation is modest, on the same order of magnitude as performing a particleto-grid transfer; this additional cost is dominated by generating the isocontour and sample points where surface tension forces are applied. Importantly, we stress that sample point generation occurs only once per time step whether explicit or implicit MPM is used. Thus, we observe that when using implicit MPM (which typically takes much larger time steps than explicit), the marginal cost of our surface tension model is quite small, anywhere from $2-53 \%$ of the cost of particle-to-grid transfers performed during a time step.

Implicit vs. Explicit Performance. While Table 2 suggests that implicit MPM involves more computationally expensive time steps, we stress that implicit MPM is able to take stable time steps orders
Table 2. Performance measurements for one time step of several of our examples, broken down by (1) generating the sample points for evaluating surface tension forces, (2) transfer of MPM particle quantities to the background grid, and (3) transfer of background grid quantities to MPM particles. All times are in milliseconds. Asterisks denote that implicit MPM was used.

\begin{tabular}{llllll}
\hline Example & \# Cells & \# Part. & Sampling & Part. $\rightarrow$ Grid & Grid $\rightarrow$ Part. \\
\hline 2D Disks $\left(k^{\sigma}=5.0\right)$ & $4 \mathrm{~K}$ & $6 \mathrm{~K}$ & 1 & 3 & 0.3 \\
Platforms $\left(k^{\sigma}=5.0\right)$ & $524 \mathrm{~K}$ & $100 \mathrm{~K}$ & 82 & 22 & 7 \\
${ }^{*}$ Ellipsoid $\left(k^{\sigma}=1.0\right)$ & $262 \mathrm{~K}$ & $208 \mathrm{~K}$ & 60 & 1723 & 5 \\
${ }^{*}$ Cube & $2.1 \mathrm{M}$ & $194 \mathrm{~K}$ & 310 & 135 & 4 \\
${ }^{*}$ Cloth & $262 \mathrm{~K}$ & $866 \mathrm{~K}$ & 146 & 4956 & 22 \\
${ }^{*}$ Moderate Droplet & $2.1 \mathrm{M}$ & $558 \mathrm{k}$ & 321 & 5635 & 16 \\
\hline
\end{tabular}

of magnitude larger than explicit MPM, and hence our fully implicit formulation can be much more performant than an explicit formulation needing impractically small $\Delta t$. For instance, in the 3D ellipsoid example, we found that modifying the surface tension coefficient to $k^{\sigma}=100$ and the bulk modulus to $83,333.33$ required a maximum $\Delta t$ of $5 \times 10^{-6} \mathrm{~s}$ for explicit MPM in order to remain stable, while implicit MPM was able to achieve stability with a maximum $\Delta t$ of $1 \times 10^{-2}$ s. Hence we measured an approximate $35 \mathrm{x}$ speedup of implicit MPM over explicit MPM in this scenario, demonstrating the importance of an implicit formulation for extremely stiff problems.

Rendering. Rendering of particle-based fluids is typically achieved by using a surfacing algorithm. A common surfacing strategy is to union spheres placed around each fluid particle, rasterize a corresponding level set to a background grid, and then form an explicit mesh of the associated zero isocontour (subsequently performing any necessary postprocesses). We note that, conveniently, this computation is highly similar to our generation of massless surface tension particle locations at simulation time. We found that for several of our examples, surfacing the sparse (codimension-one) massless particles rather than the spatially dense fluid particles allowed for not only faster rendering, but also smoother and more coherent surfaces.

Table 3. Stability across different time steps and spatial resolutions $\left(\Delta x^{-1}=\right.$ 32,128 and 512) for the example from Figure 8. Here, red squares correspond to unstable simulations, green circles correspond to stable ones, and orange triangles indicate that artifacts (such as spurious currents) were significant. We see that explicit is unstable across this whole range. While semi-implicit performs better than explicit, most of the simulations exhibited significant artifacts compared to implicit (see Figure 8).

\begin{tabular}{|c|c|c|c|c|c|c|c|c|c|}
\hline \multirow[b]{2}{*}{$\Delta t$} & \multicolumn{3}{|c|}{ Explicit } & \multicolumn{3}{|c|}{ Semi-Implicit } & \multicolumn{3}{|c|}{ Implicit } \\
\hline & 32 & 128 & 512 & 32 & 128 & 512 & 32 & 128 & 512 \\
\hline .01 & $\mathbf{\square}$ & $\square$ & $\mathbf{\square}$ & $\Delta$ & $\Delta$ & $\square$ & ○ & ○ & 0 \\
\hline .0075 & $\mathbf{\square}$ & $\mathbf{\square}$ & $\mathbf{\square}$ & $\Delta$ & $\Delta$ & 0 & ○ & ○ & 0 \\
\hline .005 & 口 & 口 & $\mathbf{\square}$ & $\boldsymbol{\Delta}$ & $\mathbf{\Delta}$ & ○ & ○ & - & 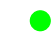 \\
\hline .0025 & $\mathbf{\square}$ & $\square$ & $\mathbf{\square}$ & $\Delta$ & $\Delta$ & 0 & - & - & 0 \\
\hline .001 & 口 & $\mathbf{\square}$ & 口 & $\Delta$ & $\Delta$ & 0 & 0 & 0 & 0 \\
\hline
\end{tabular}

ACM Trans. Graph., Vol. 39, No. 6, Article 183. Publication date: December 2020. 


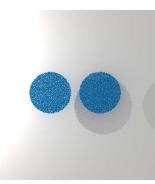

(a) Frame 1

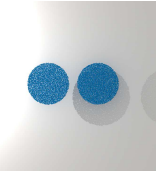

(f) Frame 1

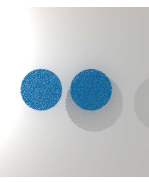

(k) Frame 1

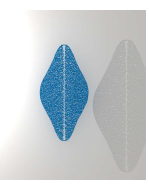

(b) Frame 12

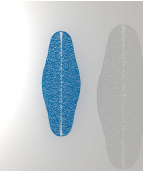

(g) Frame 12

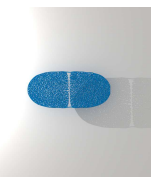

(I) Frame 12

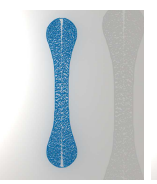

(c) Frame 32

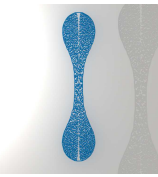

(h) Frame 32

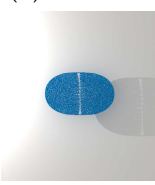

(m) Frame 32

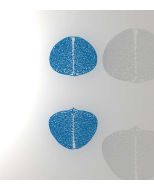

(d) Frame 86

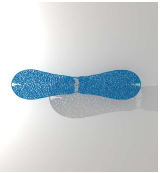

(i) Frame 86

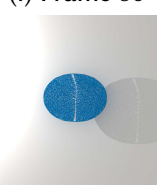

(n) Frame 86

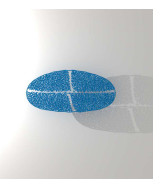

(e) Frame 150
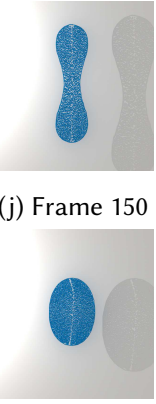

(o) Frame 150 (j) Frame 150

Fig. 9. A pair of two-dimensional circular disks collides. (Top row) With less surface tension $\left(k^{\sigma}=0.05\right)$, the disks collide, separate, then merge again, maintaining smooth interfaces while undergoing complex topological changes. (Middle row) Under slightly stronger surface tension $\left(k^{\sigma}=0.1\right)$, the disks combine but do not separate, instead exhibiting oscillatory stretching behavior. (Bottom row) With much stronger surface tension forces $\left(k^{\sigma}=5\right)$, the disks collide and rapidly oscillate while remaining stable.

\section{LIMITATIONS AND DISCUSSION}

Instead of being fully incompressible, our formulation is based on the compressible Euler equations with a penalty on compression. An incompressible formulation would require the solution of a nonlinear KKT system, which would take more time to solve but may still be preferable. Additionally, our present Newton system for the implicit formulation is solved using conjugate gradient with no preconditioner. This does not present a hurdle for solving the system, as in practice we require few CG iterations per Newton step. Nonetheless, the application of a good preconditioner could improve convergence of the CG iteration and reduce the solve time for each step.

Both our MPM discretization and our level-set based boundary sampling technique are conceptually simple and hence relatively easy to implement in contrast to most front tracking or unstructured discretizations which require dynamic remeshing. However, such approaches are more capable of maintaining sharp interfaces and are likely more accurate than ours as a result. Moreover, we note that in our current implementation surface tension forces may still exist when the liquid is in contact with a solid boundary since forces are simply defined at the zero isocontour of the level set; this may be inaccurate, and a careful treatment of adhesion forces at solid-fluid boundaries is interesting future work.

We also note that it would be trivial to incorporate a viscosity model, e.g. from [Ram et al. 2015], as future work. Furthermore, we are interested in supporting multiple interacting materials with different surface tension coefficients, such as the water droplet falling on a hydrophilic or hydrophobic surface in Clausen et al [2013]. For example, one could imagine generating level sets around

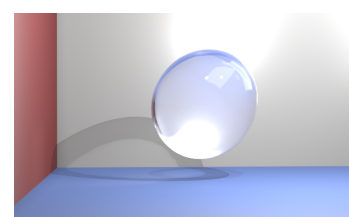

(a) Frame 7

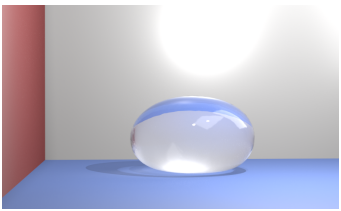

(c) Frame 9

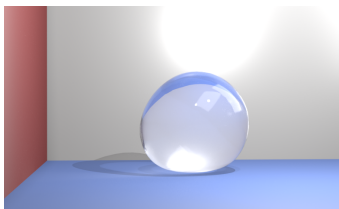

(e) Frame 10

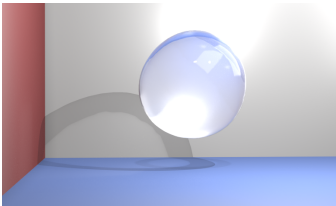

(g) Frame 12

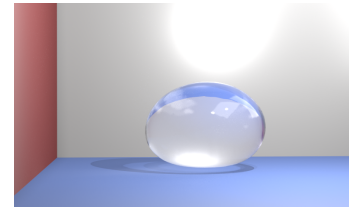

(i) Frame 23

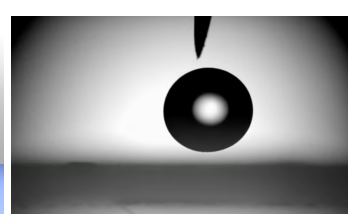

(b)

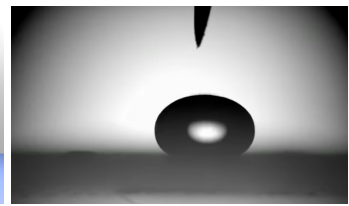

(d)

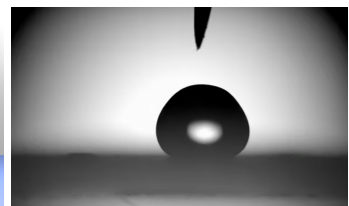

(f)

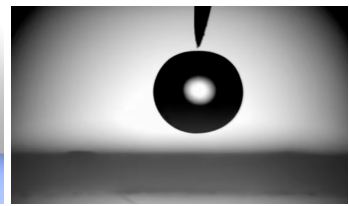

(h)

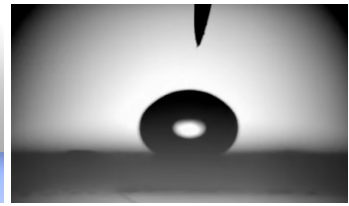

(j)
Fig. 10. (Left) A spherical droplet with very high surface tension coefficient $\left(k^{\sigma}=20\right)$ bounces multiple times off a surface. (Right) Comparable photos from experiment in [Weisensee et al. 2016] (images used with permission of author; images $\odot$ Patricia B. Weisensee et al.). Our simulation achieves qualitatively similar behavior despite the challenging surface tension coefficient.

each material and adjusting the effective surface tension coefficient for boundary particles deemed to lie along multi-material interfaces.

\section{ACKNOWLEDGMENTS}

This research was supported by the Exascale Computing Project (17SC-20-SC), a collaborative effort of the U.S. Department of Energy Office of Science and the National Nuclear Security Administration. This research was also supported under DoE ORNL contract 4000171342 .

\section{REFERENCES}

A. Adamson and A. Gast. 1967. Physical chemistry of surfaces. Vol. 150. Interscience Publishers New York.

N. Akinci, G. Akinci, and M. Teschner. 2013. Versatile surface tension and adhesion for SPH fluids. ACM Trans Graph (TOG) 32, 6 (2013), 1-8. 


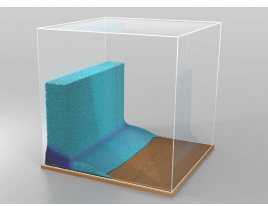

(a) Frame 7

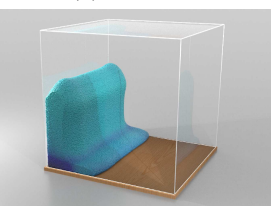

(d) Frame 7

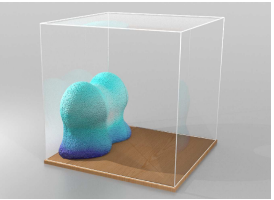

(g) Frame 7

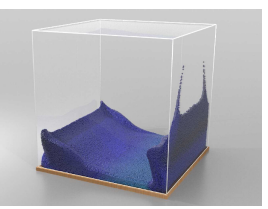

(b) Frame 25

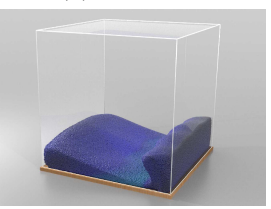

(e) Frame 25

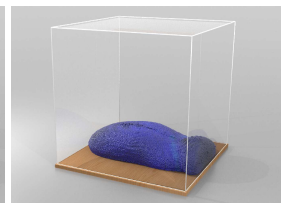

(h) Frame 25

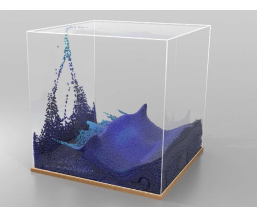

(c) Frame 40

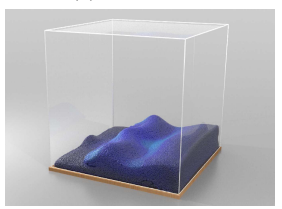

(f) Frame 40

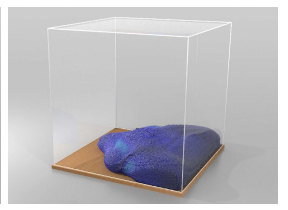

(i) Frame 40
Fig. 11. A block of fluid collapses and splashes in a tank. (Top) $k^{\sigma}=0.001$. (Middle) $k^{\sigma}=10$. (Bottom) $k^{\sigma}=100$. At low to moderate surface tension, dy namic splashing and turbulent behaviors are observed. Particles are colored by speed from blue (slow) to white (fast).

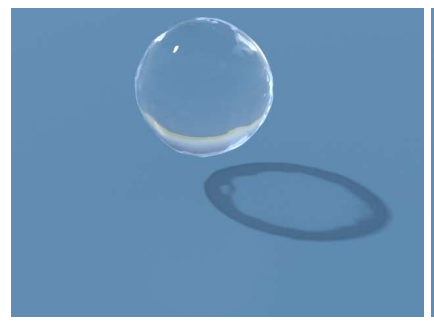

(a) Frame 9

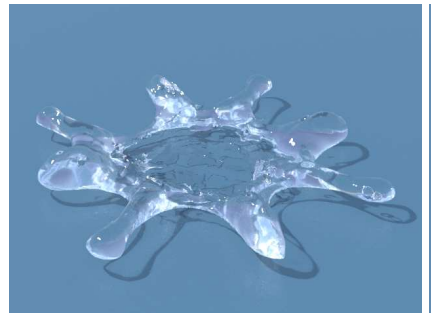

(c) Frame 15

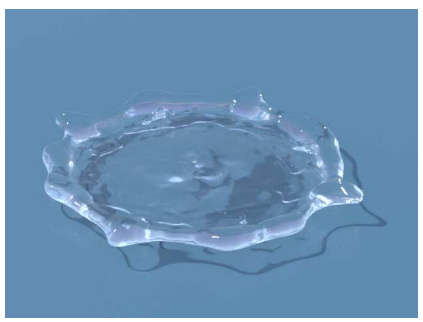

(b) Frame 12

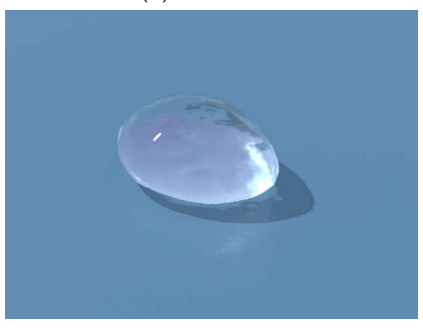

(d) Frame 60
Fig. 12. A droplet of water with moderate surface energy $\left(k^{\sigma}=0.5\right)$ hits the ground and forms interesting ring and offshoot formations before contracting back into a solid droplet, similar to the example in [Da et al. 2016].

R. Ando, N. Thurey, and C. Wojtan. 2013. Highly adaptive liquid simulations on tetrahedral meshes. ACM Trans Graph 32, 4 (2013), 103:1-103:10.

O. Azencot, O. Vantzos, M. Wardetzky, M. Rumpf, and M. Ben-Chen. 2015. Functional thin films on surfaces. In Proc 14th ACM SIGGRAPH/Eurograph Symp Comp Anim. 137-146.

E. Bänsch. 2001. Finite element discretization of the Navier-Stokes equations with a free capillary surface. Num Math 88, 2 (2001), 203-235.

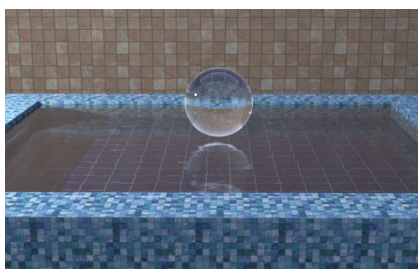

(a) Frame 0

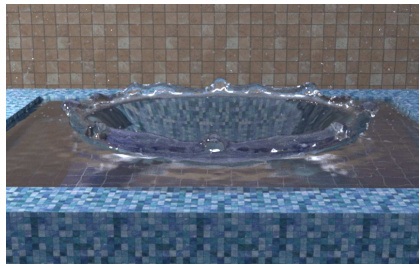

(c) Frame 11

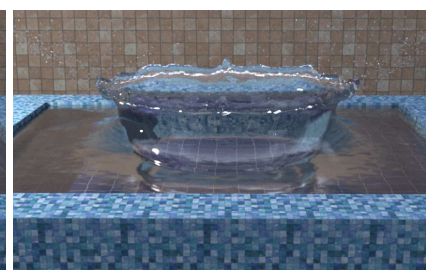

(b) Frame 7

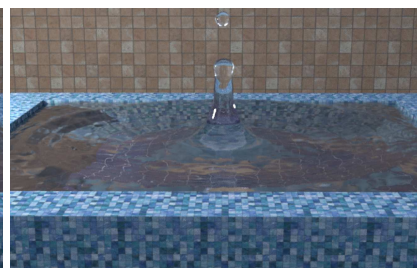

(d) Frame 17
Fig. 13. A droplet impacts a static fluid, both with $k^{\sigma}=0.075$.

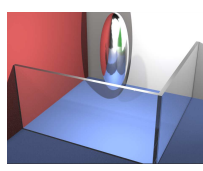

(a) Frame 1

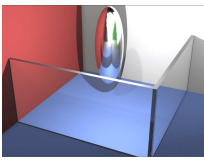

(e) Frame 1

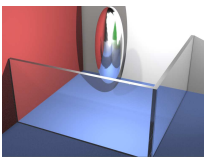

(i) Frame 1

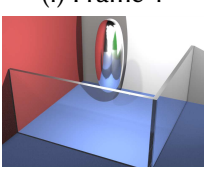

(m) Frame 1

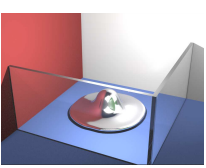

(b) Frame 10

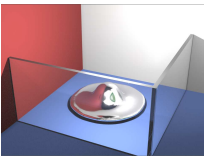

(f) Frame 10

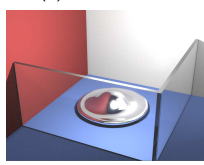

(j) Frame 10

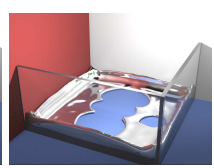

(c) Frame 17

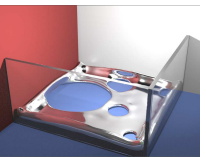

(g) Frame 17

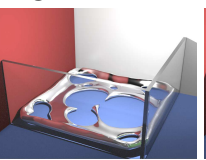

(k) Frame 17

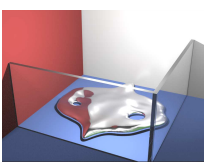

(d) Frame 49

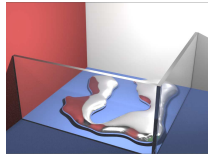

(h) Frame 49

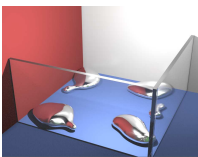

(I) Frame 49
Fig. 14. An ellipsoid of liquid metal with various values of $k^{\sigma}$ falls in a box. With less surface energy, the fluid exhibits more drastic topology changes and greater motion, while with large surface energy, the fluid remains a single droplet and rapidly oscillates. (Top row) $k^{\sigma}=0.025$. (Second row) $k^{\sigma}=0.05$. (Third row) $k^{\sigma}=0.1$. (Bottom row) $k^{\sigma}=1.0$.

C. Batty, A. Uribe, B. Audoly, and E. Grinspun. 2012. Discrete viscous sheets. ACM Trans Graph (TOG) 31, 4 (2012), 1-7.

C. Batty, S. Xenos, and B. Houston. 2010. Tetrahedral embedded boundary methods for accurate and flexible adaptive fluids. In Comp Graph For, Vol. 29. Wiley Online Library, 695-704.

M. Becker and M. Teschner. 2007. Weakly Compressible SPH for Free Surface Flows. In Proc ACM SIGGRAPH/Eurograph Symp Comp Anim. 209-217.

T. Belytschko, W. Liu, B. Moran, and K. Elkhodary. 2013. Nonlinear finite elements for continua and structures. John Wiley and sons. 


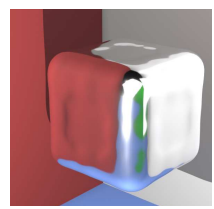

(a) Frame 2

(e) Frame 2

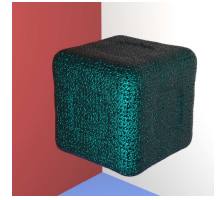

(i) Frame 2

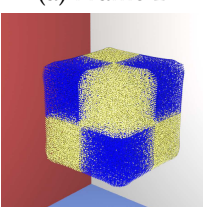

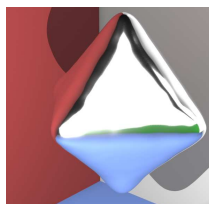

(b) Frame 41

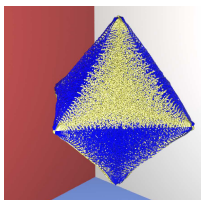

(f) Frame 41

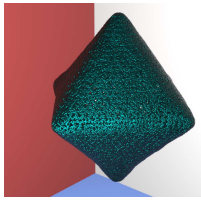

(j) Frame 41

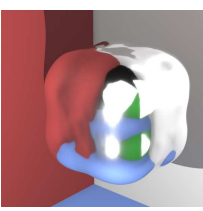

(c) Frame 80

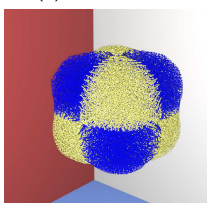

(g) Frame 80

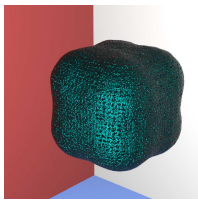

(k) Frame 80

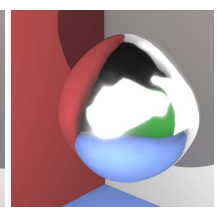

(d) Frame 240

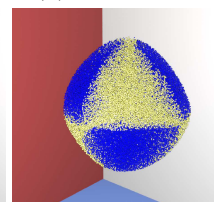

(h) Frame 240

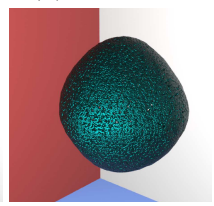

(I) Frame 240
Fig. 15. A cubic fluid relaxes under surface tension forces with no gravity The geometry oscillates between a cube and an octrahedral shape a number of times, and eventually tends towards a spherical steady-state as energy is lost numerically. The middle row shows the fluid particles and the bottom row shows the massless boundary sampling particles.

J. Bonet and R. Wood. 2008. Nonlinear continuum mechanics for finite element analysis. Cambridge University Press.

L. Boyd and R. Bridson. 2012. MultiFLIP for energetic two-phase fluid simulation. ACM Trans Graph 31, 2 (2012), 16:1-16:12

J. Brackbill, D. Kothe, and C. Zemach. 1992. A continuum method for modeling surface tension. 7 Comp Phys 100, 2 (1992), 335-354.

R. Bridson. 2008. Fluid simulation for computer graphics. Taylor \& Francis.

T. Brochu, C. Batty, and R. Bridson. 2010. Matching fluid simulation elements to surface geometry and topology. ACM Trans Graph 29, 4 (2010), 47:1-47:9.

T. Brochu and R. Bridson. 2009. Robust topological operations for dynamic explicit surfaces. SIAM 7 Sci Comp 31, 4 (2009), 2472-2493. https://doi.org/10.1137/080737617

G. Buscaglia and R. Ausas. 2011. Variational formulations for surface tension, capillarity and wetting. Comp Meth App Mech Eng 200, 45-46 (2011), 3011-3025.

P. Clausen, M. Wicke, J. R. Shewchuk, and J. F. O'brien. 2013. Simulating liquids and solid-liquid interactions with Lagrangian meshes. ACM Transactions on Graphics (TOG) 32, 2 (2013), 17.

S. Clavet, P. Beaudoin, and P. Poulin. 2005. Particle-based viscoelastic fluid simulation In Proc 2005 ACM SIGGRAPH/Eurograph Symp Comp Anim. 219-228.

D. Cohen-Steiner and J. Morvan. 2003. Restricted delaunay triangulations and normal cycle. In Proc 19th Comp Geometry. 312-321.

F. Da, C. Batty, and E. Grinspun. 2014. Multimaterial mesh-based surface tracking. ACM Trans Graph 33, 4 (2014), 112:1-112:11. https://doi.org/10.1145/2601097.2601146

F. Da, C. Batty, C. Wojtan, and E. Grinspun. 2015. Double bubbles sans toil and trouble discrete circulation-preserving vortex sheets for soap films and foams. ACM Trans Graph (SIGGRAPH 2015) (2015).

F. Da, D. Hahn, C. Batty, C. Wojtan, and E. Grinspun. 2016. Surface-only liquids. ACM Trans Graph (TOG) 35, 4 (2016), 1-12.

F. de Goes, C. Wallez, J. Huang, D. Pavlov, and M. Desbrun. 2015. Power particles an incompressible fluid solver based on power diagrams. ACM Trans. Graph. 34, 4 (2015), 50-1.

F. Denner and B. van Wachem. 2015. Numerical time-step restrictions as a result of capillary waves. 7 Comp Phys 285 (2015), 24-40.

G. Dilts. 2000. Moving least-squares particle hydrodynamics II: conservation and boundaries. Int 7 Num Meth Eng 48, 10 (2000), 1503-1524.

I. Eckstein, J. Pons, Y. Tong, C. Kuo, and M. Desbrun. 2007. Generalized surface flows for mesh processing. In Proc Eurograph Symp Geom Proc. Eurographics Association, 183-192.

D. Enright, R. Fedkiw, J. Ferziger, and I. Mitchell. 2002. A Hybrid Particle Level Set Method for Improved Interface Capturing. 7. Comput. Phys. 183, 1 (2002), 83-116.
D. Enright, D. Nguyen, F. Gibou, and R. Fedkiw. 2003. Using the particle level set method and a second order accurate pressure boundary condition for free surface flows. In ASME/FSME 2003 4th foint Fluids Summer Engineering Conference. ASMEDC, 337-342.

Y. Fei, C. Batty, E. Grinspun, and C. Zheng. 2018. A multi-scale model for simulating liquid-fabric interactions. ACM Trans Graph 37, 4 (2018), 51:1-51:16. https://doi. org $/ 10.1145 / 3197517.3201392$

T. Gast, C. Schroeder, A. Stomakhin, C. Jiang, and J. Teran. 2015. Optimization Integrator for Large Time Steps. IEEE Trans Vis Comp Graph 21, 10 (2015), 1103-1115.

F. Gibou, R. P. Fedkiw, L.-T. Cheng, and M. Kang. 2002. A Second-Order-Accurate Symmetric Discretization of the Poisson Equation on Irregular Domains. F. Comput. Phys. 176, 1 (2002), 205-227.

O. Gonzalez and A. Stuart. 2008. A first course in continuum mechanics. Cambridge University Press.

A. Haque and G. Dilts. 2007. Three-dimensional boundary detection for particle methods. 7 Comp Phys 226, 2 (2007), 1710-1730.

F. Harlow. 1964. The particle-in-cell method for numerical solution of problems in fluid dynamics. Meth Comp Phys 3 (1964), 319-343.

X. He, N. Liu, G. Wang, F. Zhang, S. Li, S. Shao, and H. Wang. 2012. Staggered meshless solid-fluid coupling. ACM Trans Graph (TOG) 31, 6 (2012), 1-12.

J. Hochstein and T. Williams. 1996. An implicit surface tension model. In 34th Aerospace Sciences Meeting and Exhibit. 599.

T. Hou, J. Lowengrub, and M. Shelley. 1994. Removing the stiffness from interfacial flows with surface tension. J Comp Phys 114, 2 (1994), 312-338.

D.A.B. Hyde, S.W. Gagniere, A. Marquez-Razon, and J. Teran. 2020. Supplementary Technical Document. Technical Report.

S. Hysing. 2006. A new implicit surface tension implementation for interfacial flows. Int 7 Num Meth Fl 51, 6 (2006), 659-672.

A. Jarauta, P. Ryzhakov, J. Pons-Prats, and M. Secanell. 2018. An implicit surface tension model for the analysis of droplet dynamics. F Comp Phys 374 (2018), 1196-1218.

C. Jiang, C. Schroeder, A. Selle, J. Teran, and A. Stomakhin. 2015. The Affine ParticleIn-Cell Method. ACM Trans Graph 34, 4 (2015), 51:1-51:10.

C. Jiang, C. Schroeder, J. Teran, A. Stomakhin, and A. Selle. 2016. The Material Point Method for Simulating Continuum Materials. In ACM SIGGRAPH 2016 Course. 24:124:52.

T. Kim, F. De Goes, and H. Iben. 2019. Anisotropic elasticity for inversion-safety and element rehabilitation. ACM Trans Graph (TOG) 38, 4 (2019), 1-15.

W. Li, D. Liu, M. Desbrun, J. Huang, and X. Liu. 2020. Kinetic-based Multiphase Flow Simulation. IEEE Trans Vis Comp Graph (2020).

O. Mercier, C. Beauchemin, N. Thuerey, T. Kim, and D. Nowrouzezahrai. 2015. Surface turbulence for particle-based liquid simulations. ACM Trans Graph 34(6) (Nov 2015), 10.

M. Misztal and J. Bærentzen. 2012. Topology-adaptive interface tracking using the deformable simplicial complex. ACM Trans Graph (TOG) 31, 3 (2012), 1-12.

M. Misztal, K. Erleben, A. Bargteil, J. Fursund, B. Christensen, J. Bærentzen, and R. Bridson. 2013. Multiphase flow of immiscible fluids on unstructured moving meshes. IEEE Trans Vis Comp Graph 20, 1 (2013), 4-16.

J. Morris. 2000. Simulating surface tension with smoothed particle hydrodynamics. Int 7 Num Meth Fl 33, 3 (2000), 333-353.

M. Müller. 2009. Fast and robust tracking of fluid surfaces. In Proc ACM SIGGRAPH/Eurograph Symp Comp Anim. ACM, 237-245. https://doi.org/10.1145/ 1599470.1599501

M. Müller, D. Charypar, and M. Gross. 2003. Particle-based fluid simulation for interactive applications. In Proc 2003 ACM SIGGRAPH/Eurograph Symp Comp Anim. Eurographics Association, 154-159.

J. Orthmann, H. Hochstetter, B. Julian J. Bader, S. Bayraktar, and A. Kolb. 2013 Consistent surface model for SPH-based fluid transport. In Proc 12th ACM SIGGRAPH/Eurograph Symp Comp Anim. 95-103.

T. Patterson, N. Mitchell, and E. Sifakis. 2012. Simulation of complex nonlinear elastic bodies using lattice deformers. ACM Trans Graph (TOG) 31, 6 (2012), 1-10.

S. Popinet. 2018. Numerical models of surface tension. Annual Rev Fluid Mech 50 (2018), $49-75$

D. Ram, T. Gast, C. Jiang, C. Schroeder, A. Stomakhin, J. Teran, and P. Kavehpour. 2015 A material point method for viscoelastic fluids, foams and sponges. In Proc ACM SIGGRAPH/Eurograph Symp Comp Anim. 157-163.

M. Sandim, D. Cedrim, L. Nonato, P. Pagliosa, and A. Paiva. 2016. Boundary detection in particle-based fluids. Comp Graph For 35, 2 (2016), 215-224. https://doi.org/10 1111/cgf.12824

C. Schroeder, W. Zheng, and R. Fedkiw. 2012. Semi-implicit surface tension formulation with a Lagrangian surface mesh on an Eulerian simulation grid. 7 Comp Phys 231, 4 (2012), 2092-2115.

E. Sifakis and J. Barbic. 2012. FEM simulation of 3D deformable solids: a practitioner's guide to theory, discretization and model reduction. In ACM SIGGRAPH 2012 Courses (Los Angeles, California) (SIGGRAPH '12). ACM, New York, NY, USA, 20:1-20:50. https://doi.org/10.1145/2343483.2343501 


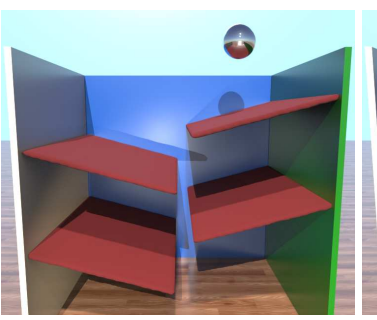

(a) Frame 1

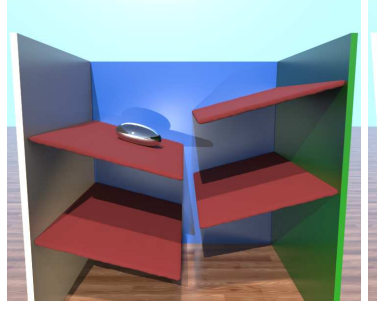

(e) Frame 37

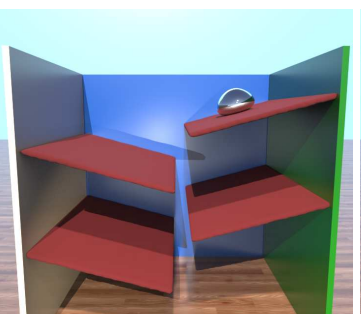

(b) Frame 7

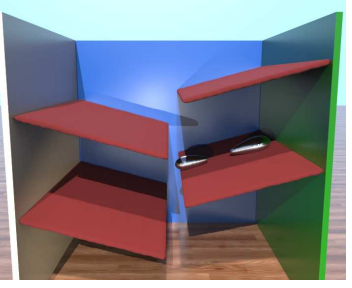

(f) Frame 60

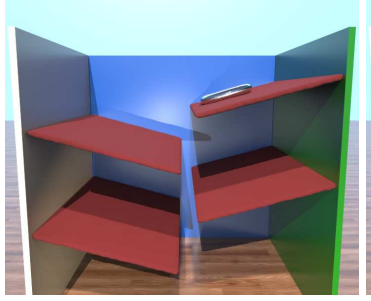

(c) Frame 10

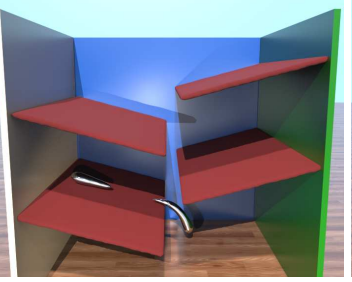

(g) Frame 81

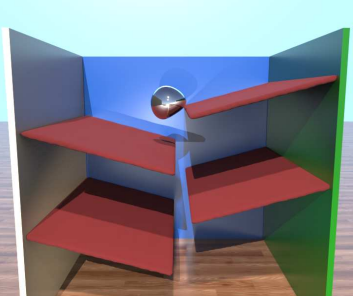

(d) Frame 16

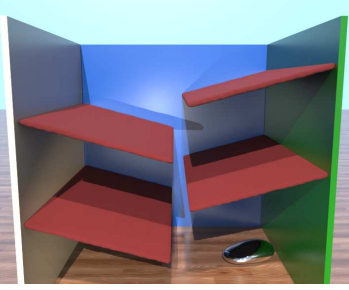

(h) Frame 106

Fig. 16. A spherical liquid metal droplet with $k^{\sigma}=5.0$ exhibits one-way coupled behavior as it falls on a series of fixed platforms in a rectangular domain. The droplet separates in two and re-merges several times as it falls.

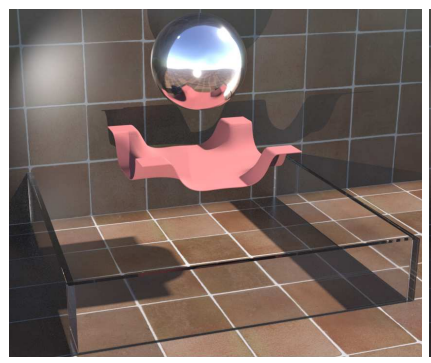

(a) Frame 4

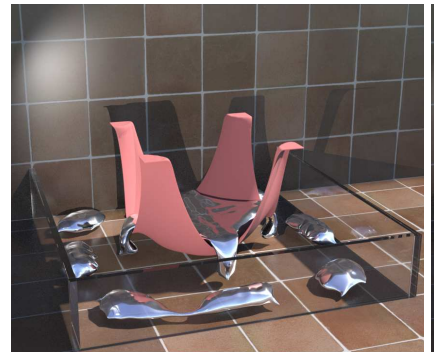

(c) Frame 44

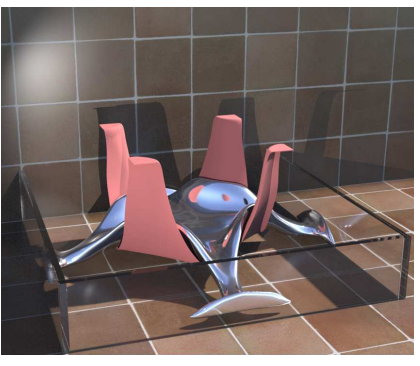

(b) Frame 15

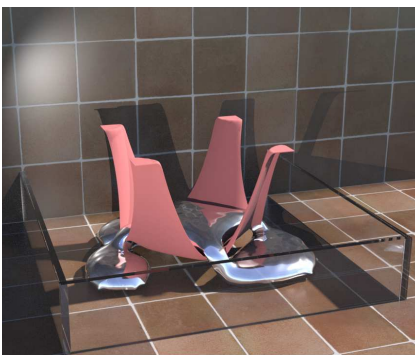

(d) Frame 131
Fig. 17. A spherical fluid droplet $\left(k^{\sigma}=1.0\right)$ falls on a cloth sheet with two-way coupling between the fluid and sheet. The droplet rolls off the sheet and splits into several disjoint components, which tend to re-merge as the simulation progresses and the cloth sinks under the weight of the fluid.

F. Sin, A. Bargteil, and J. Hodgins. 2009. A point-based method for animating incompressible flow. In Proc 2009 ACM SIGGRAPH/Eurographics Symp Comp Anim. 247-255.

B. Smith, F. Goes, and T. Kim. 2019. Analytic Eigensystems for Isotropic Distortion Energies. ACM Trans Graph (TOG) 38, 1 (2019), 1-15.

A. Stomakhin, C. Schroeder, L. Chai, J. Teran, and A. Selle. 2013. A Material Point Method for snow simulation. ACM Trans Graph 32, 4 (2013), 102:1-102:10.
D. Sulsky, Z. Chen, and H. Schreyer. 1994. A particle method for history-dependent materials. Comp Meth App Mech Eng 118, 1 (1994), 179-196.

M. Sussman and M. Ohta. 2009. A stable and efficient method for treating surface tension in incompressible two-phase flow. SIAM 7 Sci Comp 31, 4 (2009), 2447-2471.

J. Teran, E. Sifakis, G. Irving, and R. Fedkiw. 2005. Robust quasistatic finite elements and flesh simulation. In Proc 2005 ACM SIGGRAPH/Eurograph Symp Comp Anim. 181-190.

N. Thürey, C. Wojtan, M. Gross, and G. Turk. 2010. A multiscale approach to mesh-based surface tension flows. ACM Trans Graph (TOG) 29, 4 (2010), 1-10.

X. Wang, M. Li, Y. Fang, X. Zhang, M. Gao, M. Tang, D. Kaufman, and C. Jiang. 2020. Hierarchical optimization time integration for CFL-rate MPM stepping. ACM Trans Graph (TOG) 39, 3 (2020), 1-16.

P. Weisensee, J. Tian, N. Miljkovic, and W. King. 2016. Water droplet impact on elastic superhydrophobic surfaces. Scientific Reports 6 (2016). Issue 1. https://doi.org/10. 1038/srep30328

M. Wicke, D. Ritchie, B. Klingner, S. Burke, J. Shewchuk, and J. O’Brien. 2010. Dynamic local remeshing for elastoplastic simulation. ACM Trans Graph 29, 4 (2010), 49:1-11.

C. Wojtan, N. Thürey, M. Gross, and G. Turk. 2010. Physics-inspired topology changes for thin fluid features. ACM Trans Graph 29, 4 (2010), 50:1-50:8. https://doi.org/10. $1145 / 1778765.1778787$

C. Wojtan and G. Turk. 2008. Fast viscoelastic behavior with thin features. ACM Trans Graph 27, 3 (2008), 1-8. https://doi.org/10.1145/1360612.1360646

S. Yang, X. He, H. Wang, S. Li, G. Wang, E. Wu, and K. Zhou. 2016. Enriching SPH simulation by approximate capillary waves. In Symp Comp Anim. 29-36.

J. Yu, C. Wojtan, G. Turk, and C. Yap. 2012. Explicit mesh surfaces for particle based fluids. Comp Graph Forum 31, 2 pt4 (2012), 815-824. https://doi.org/10.1111/j.14678659.2012.03062.x

Y. Zhang, B. Solenthaler, and R. Pajarola. 2008. Adaptive sampling and rendering of fluids on the GPU. In Proc Symp Point-Based Graph. 137-146. https://doi.org/10.5167/uzh9735

X. Zhao, S. Xu, and J. Liu. 2017. Surface tension of liquid metal: role, mechanism and application. Front Energy 11, 4 (2017), 535-567.

W. Zheng, B. Zhu, B. Kim, and R. Fedkiw. 2015. A new incompressibility discretization for a hybrid particle MAC grid representation with surface tension. 7 Comp Phys 280 (2015), 96-142.

B. Zhu, E. Quigley, M. Cong, J. Solomon, and R. Fedkiw. 2014. Codimensional surface tension flow on simplicial complexes. ACM Trans Graph (TOG) 33, 4 (2014), 1-11.

F. Zorilla, M. Ritter, J. Sappl, W. Rauch, and M. Harders. 2020. Accelerating surface tension calculation in SPH via particle classification and Monte Carlo integration. Computers 9, 2 (2020), 23. 